NBER WORKING PAPER SERIES

\title{
PRACTICAL VOLATILITY AND CORRELATION MODELING FOR FINANCIAL MARKET RISK MANAGEMENT
}

\author{
Torben G. Andersen \\ Tim Bollerslev \\ Peter F. Christoffersen \\ Francis X. Diebold \\ Working Paper 11069 \\ http://www.nber.org/papers/w11069 \\ NATIONAL BUREAU OF ECONOMIC RESEARCH \\ 1050 Massachusetts Avenue \\ Cambridge, MA 02138 \\ January 2005
}

This paper is prepared for Mark Carey and René Stulz (eds.), Risks of Financial Institutions, University of Chicago Press for NBER. For helpful comments we would like to thank Ken Abbott, Casper de Vries, Philipp Hartmann, Patricia Jackson, Jim O'Brien, Hashem Pesaran, and Pedro Santa-Clara. For research support, Andersen, Bollerslev and Diebold thank the U.S. National Science Foundation, and Christoffersen thanks FQRSC, SSHRC and IFM2. The views expressed herein are those of the author(s) and do not necessarily reflect the views of the National Bureau of Economic Research.

(C) 2005 by Torben G. Andersen, Tim Bollerslev, Peter F. Christoffersen, and Francis X. Diebold. All rights reserved. Short sections of text, not to exceed two paragraphs, may be quoted without explicit permission provided that full credit, including () notice, is given to the source. 
Practical Volatility and Correlation Modeling for Financial Market Risk Management Torben G. Andersen, Tim Bollerslev, Peter F. Christoffersen, and Francis X. Diebold NBER Working Paper No. 11069

January 2005

JEL No. G1

\begin{abstract}
$\underline{\text { ABSTRACT }}$
What do academics have to offer market risk management practitioners in financial institutions? Current industry practice largely follows one of two extremely restrictive approaches: historical simulation or RiskMetrics. In contrast, we favor flexible methods based on recent developments in financial econometrics, which are likely to produce more accurate assessments of market risk. Clearly, the demands of real-world risk management in financial institutions - in particular, realtime risk tracking in very high-dimensional situations - impose strict limits on model complexity. Hence we stress parsimonious models that are easily estimated, and we discuss a variety of practical approaches for high-dimensional covariance matrix modeling, along with what we see as some of the pitfalls and problems in current practice. In so doing we hope to encourage further dialog between the academic and practitioner communities, hopefully stimulating the development of improved market risk management technologies that draw on the best of both worlds.
\end{abstract}

Torben G. Andersen

Department of Finance

Kellogg School of Management

Northwestern University

Evanston, IL 60208

and NBER

t-andersen@kellogg.northwestern.edu

Tim Bollerslev

Department of Economics

Duke University

Durham, NC 27708

and NBER

boller@econ.duke.edu
Peter F. Christoffersen

Faculty of Management

McGill University

Montreal, Quebec H3A 1G5

peter.christoffersen@mcgill.ca

Francis X. Diebold

Department of Economics

University of Pennsylvania

Philadelphia, PA 19104

and NBER

fdiebold@sas.upenn.edu 


\section{Introduction}

It is now widely agreed that financial asset return volatilities and correlations (henceforth "volatilities") are time-varying, with persistent dynamics. This is true across assets, asset classes, time periods, and countries. Moreover, asset return volatilities are central to finance, whether in asset pricing, portfolio allocation, or market risk measurement. Hence the field of financial econometrics devotes considerable attention to time-varying volatility and associated tools for its measurement, modeling and forecasting.

Here we survey, unify and extend recent developments in the financial econometrics of time-varying volatility, focusing exclusively on practical applications to the measurement and management of market risk, stressing parsimonious models that are easily estimated. Our ultimate goal is to stimulate dialog between the academic and practitioner communities, advancing best-practice market risk measurement and management technologies by drawing upon the best of both worlds. Three themes appear repeatedly, and so we highlight them here.

The first is the issue of aggregation level. We consider both aggregated (portfolio level) and disaggregated (asset level) modeling, emphasizing the related distinction between risk measurement and risk management, because risk measurement generally requires only a portfolio-level model, whereas risk management requires an asset-level model. At the asset level, the issue of dimensionality and dimensionality reduction arises repeatedly, and we devote considerable attention to methods for tractable modeling of the very high-dimensional covariance matrices of practical relevance.

The second theme concerns the use of low-frequency vs. high-frequency data, and the associated issue of parametric vs. nonparametric volatility measurement. We treat all cases, but we emphasize the appeal of volatility measurement using nonparametric methods in conjunction with high-frequency data, followed by modeling that is intentionally parametric.

The third theme relates to the issue of unconditional vs. conditional risk measurement. We argue that, for most financial risk management purposes, the conditional perspective is exclusively relevant, notwithstanding, for example, the fact that popular approaches based on historical simulation and extreme-value theory typically adopt an unconditional perspective. We advocate, moreover, moving beyond a conditional volatility perspective to a full conditional density perspective, and we discuss methods for constructing and evaluating full conditional density forecasts.

We proceed systematically in several steps. In section 2, we consider portfolio level analysis, directly modeling portfolio volatility using historical simulation, exponential smoothing, and GARCH methods. In section 3, we consider asset level analysis, modeling asset covariance matrices using exponential smoothing and multivariate GARCH methods, paying special attention to dimensionality-reduction methods. In section 4, we explore the use of highfrequency data for improved covariance matrix measurement and modeling, treating realized variance and covariance, and again discussing procedures for dimensionality reduction. In section 5 we treat the construction of complete conditional density forecasts via simulation methods. We conclude in section 6 . 


\section{Portfolio Level Analysis: Modeling Portfolio Volatility}

Portfolio risk measurement requires only a univariate, portfolio-level model (e.g., Benson and Zangari, 1997). In this section we discuss such univariate, portfolio-based methods. In contrast, active portfolio risk management, including portfolio allocation, requires a multivariate model, as we discuss subsequently in section 3 .

In particular, portfolio level analysis is rarely done other than via historical simulation (defined below). But we will argue that there is no reason why one cannot estimate a parsimonious dynamic model for portfolio level returns. If interest centers on the distribution of the portfolio returns, then this distribution can be modeled directly rather than via aggregation based on a larger and almost inevitably less-well-specified multivariate model.

Berkowitz and O'Brien (2002) find evidence that existing bank risk models perform poorly and are easy outperformed by a simple univariate GARCH model (defined below). Their result is remarkable in that they estimate a GARCH model fit to the time series of actual historical portfolio returns where the underlying asset weights are changing over time. Berkowitz and O'Brien find that bank's reported Value-at-Risk (VaRs) on average underestimate risk when comparing ex post $\mathrm{P} / \mathrm{Ls}$ with ex ante VaR forecasts. This finding could however simply be due to the reported P/Ls being "dirty" in that they contain non-risky income from fees, commissions and intraday trading profits. ${ }^{1}$ More seriously though, Berkowitz and O'Brien find that the VaR violations which do occur tend to cluster in time. Episodes such as the Fall 1998 Russia default and LTCM debacle set off a dramatic and persistent increase in market volatility which bank models appear to largely ignore, or at least react to with considerable delay. Such VaR violation clustering is evidence of a lack of conditionality in bank VaR systems which in turn is a key theme in our discussion below. ${ }^{2}$

We first discuss the construction of historical portfolio values, which is a necessary precursor to any portfolio-level VaR analysis. We then discuss direct computation of portfolio VaR via historical simulation, exponential smoothing, and GARCH modeling. ${ }^{3}$

\subsection{Constructing Historical Pseudo Portfolio Values}

In principle it is easy to construct a time series of historical portfolio returns using current

${ }^{1}$ Even though the Basel Accord calls for banks to report 1\% VaR's, for various reasons most banks tend to actually report more conservative VaR's. Rather than simply scaling up a $1 \%$ VaR based on some "arbitrary" multiplication factor, the procedures that we discuss below is readily adapted to achieve any desired, more conservative, VaR.

${ }^{2}$ See also Jackson, Maude and Perraudin (1997).

${ }^{3}$ Duffie and Pan (1997) provide an earlier incisive discussion of related VaR procedures and corresponding practical empirical problems. 
portfolio holdings and historical asset returns:

$$
r_{w, t}=\sum_{i=1}^{N} w_{i, T} r_{i, t} \equiv W_{T}^{\prime} R_{t}, \quad t=1,2, \ldots, T .
$$

In practice, however, historical asset prices for the assets held today may not be available. Examples where difficulties arise include derivatives, individual bonds with various maturities, private equity, new public companies, merger companies and so on.

For these cases "pseudo" historical prices must be constructed using either pricing models, factor models or some ad hoc considerations. The current assets without historical prices can for example be matched to "similar" assets by capitalization, industry, leverage, and duration. Historical pseudo asset prices and returns can then be constructed using the historical prices on these substitute assets.

\section{$\underline{2.2 \text { Volatility via Historical Simulation }}$}

Banks often rely on VaRs from historical simulations (HS-VaR). In this case the VaR is calculated as the 100p'th percentile or the $(\mathrm{T}+1) \mathrm{p}$ 'th order statistic of the set of pseudo returns calculated in (1). We can write

$$
H S-V a R_{T+1 \mid T}^{p} \equiv r_{w}((T+1) p),
$$

where $r_{w}((T+1) p)$ is taken from the set of ordered pseudo returns $\left\{r_{w}(1), r_{w}(2), \ldots, r_{w}(T)\right\}$. If $(\mathrm{T}+1) \mathrm{p}$ is not an integer value then the two adjacent observations can be interpolated to calculate the VaR.

Historical simulation has some serious problems, which have been well-documented. Perhaps most importantly, it does not properly incorporate conditionality into the VaR forecast. The only source of dynamics in the HS-VaR is the fact that the sample window in (1) is updated over time. However, this source of conditionality is minor in practice. ${ }^{4}$

Figure 1 illustrates the hidden dangers of HS as discussed by Pritsker (2001). We plot the daily percentage loss on an S\&P500 portfolio along with the $1 \%$ HS-VaR calculated from a 250 day moving window. The crash on October 19, 1987 dramatically increased market volatility; however, the HS-VaR barely moves. Only after the second large drop which occurred on October 26 does the HS-VaR increase noticeably.

This admittedly extreme example illustrates a key problem with the HS-VaR.

${ }^{4}$ Bodoukh, Richardson, Whitelaw (1998) introduce updating into the historical simulation method. Note, however, the concerns in Pritsker (2001). 
Mechanically, from equation (2) we see that HS-VaR changes significantly only if the observations around the order statistic $r_{w}((T+1) p)$ change significantly. When using a 250-day moving window for a $1 \% \mathrm{HS}-\mathrm{VaR}$, only the second and third smallest returns will matter for the calculation. Including a crash in the sample, which now becomes the smallest return, may therefore not change the HS-VaR very much if the new second smallest return is similar to the previous one.

Moreover, the lack of a properly-defined conditional model in the HS methodology implies that it does not allow for the construction of a term structure of VaR. Calculating a 1\% 1day HS-VaR may be possible on a window of 250 observations, but calculating a 10-day $1 \%$ $\mathrm{VaR}$ on 250 daily returns is not. Often the 1-day VaR is simply scaled by the square root of 10 , but this extrapolation is only valid under the assumption of i.i.d. daily returns. A redeeming feature of the daily HS-VaR is exactly that it does not rely on an assumption of i.i.d. returns, and the square root scaling therefore seems curious at best.

In order to further illustrate the lack of conditionality in the HS-VaR method consider Figure 2. We first simulate daily portfolio returns from a mean-reverting volatility model and then calculate the nominal $1 \% \mathrm{HS}-\mathrm{VaR}$ on these returns using a moving window of 250 observations. As the true portfolio return distribution is known, the true daily coverage of the nominal 1\% HS-VaR can be calculated using the return generating model. Figure 2 shows the conditional coverage probability of the $1 \% \mathrm{HS}-\mathrm{VaR}$ over time. Notice from the figure how an HS-VaR with a nominal coverage probability of $1 \%$ can have a true conditional probability as high as $10 \%$, even though the unconditional coverage is correctly calibrated at $1 \%$. On any given day the risk manager thinks that there is a $1 \%$ chance of getting a return worse than the HS-VaR but in actuality there may as much as a $10 \%$ chance of exceeding the VaR. Figure 2 highlights the potential benefit of conditional density modeling: The HS-VaR computes an essentially unconditional $\mathrm{VaR}$ which on any given day can be terribly wrong. A conditional density model will generate a dynamic $\mathrm{VaR}$ in an attempt to keep the conditional coverage rate at $1 \%$ on any given day, thus creating a horizontal line in Figure 2.

The above discussion also hints at a problem with the VaR risk measures itself. It does not say anything about how large the expected loss will be on the days where the VaR is exceeded. Other measures such as expected shortfall do, but VaR has emerged as the industry risk measurement standard and we will focus on it here. The methods we will suggest below can, however, equally well be used to calculate expected shortfall and other related risk measures.

\section{$\underline{2.3 \text { Volatility via Exponential Smoothing }}$}

Although the HS-VaR methodology discussed above makes no explicit assumptions about the distributional model generating the returns, the RiskMetrics (RM) filter/model instead assumes a very tight parametric specification. One can begin to incorporate conditionality via univariate portfolio-level exponential smoothing of squared portfolio returns, in precise parallel to the exponential smoothing of individual return squares and cross products that underlies RM. 
Still taking the portfolio-level pseudo returns from (1) as the data series of interest we can define the portfolio-level RM variance as

$$
\sigma_{t}^{2}=\lambda \sigma_{t-1}^{2}+(1-\lambda) r_{w, t-1}^{2}
$$

where the variance forecast for day $\mathrm{t}$ is constructed at the end of day $\mathrm{t}-1$ using the square of the return observed at the end of day $\mathrm{t}-1$ as well as the variance on day $\mathrm{t}-1$. In practice this recursion can be initialized by setting the initial $\sigma_{0}^{2}$ equal to the unconditional sample standard deviation, say $\hat{\sigma}^{2}$.

Note that back substitution in (3) yields an expression for the current smoothed value as an exponentially weighted moving average of past squared returns:

$$
\sigma_{t}^{2}=\sum_{j=0}^{\infty} \varphi_{j} r_{w, t-1-j}^{2},
$$

where $\varphi_{j}=(1-\lambda) \lambda^{j}$. Hence the name "exponential smoothing."

Following RM, the VaR is simply calculated as

$$
R M-V a R_{T+1 \mid T}^{p} \equiv \sigma_{T+1} \Phi_{p}^{-1}
$$

where $\Phi_{\mathrm{p}}^{-1}$ denotes the pth quantile in the standard normal distribution. Although the smoothing parameter $\lambda$ may in principle be calibrated to best fit the specific historical returns at hand, following RM it is often simply fixed at 0.94 with daily returns. The implicit assumption of zero mean and standard normal innovations therefore implies that no parameters need to be estimated.

The conditional variance for the k-day aggregate return in RM is simply

$$
\operatorname{Var}\left(r_{w, t+k}+r_{w, t+k-1}+\ldots+r_{w, t+1} \mid \mathscr{F}_{t}\right) \equiv \sigma_{t: t+k \mid t}^{2}=k \sigma_{t+1}^{2} .
$$

The RM model can thus be thought of as a random walk model in variance. The lack of meanversion in the RM variance model implies that the term structure of volatility is flat. Figure 3 illustrates the difference between the volatility term structure for the random walk RM model versus a mean-reverting volatility model. Assuming a low current volatility, which is identical across models, the mean-reverting model will display an upward sloping term structure of volatility whereas the RM model will extrapolate the low current volatility across all horizons. When taken this literally the RM model does not appear to be a prudent approach to volatility modeling. The dangers of scaling the daily variance by $\mathrm{k}$, as done in (5), are discussed further in Diebold, Hickman, Inoue, and Schuermann (1998).

\section{$\underline{2.4 \text { Volatility via GARCH}}$}


The implausible temporal aggregation properties of the RM model which we discussed above motivates us to introduce the general class of GARCH models which imply meanreversion and which contain the RM model as a special case.

First we specify the general univariate portfolio return process

$$
r_{w, t}=\mu_{t}+\sigma_{t} z_{t} \quad z_{t} \sim \text { i.i.d. } \quad E\left(z_{t}\right)=0 \quad \operatorname{Var}\left(z_{t}\right)=1 .
$$

In the following we will assume that the mean is zero which is common in risk management, at least when short horizons are considered. Although difficult to estimate with much accuracy in practice, mean-dynamics could in principle easily be incorporated into the models discussed below.

The simple symmetric GARCH(1,1) model introduced by Bollerslev (1986) is written as

$$
\sigma_{t}^{2}=\omega+\alpha r_{w, t-1}^{2}+\beta \sigma_{t-1}^{2}
$$

Extensions to higher order models are straightforward, but for notational simplicity we will concentrate on the $(1,1)$ case here and throughout the chapter. Repeated substitution in (7) readily yields,

$$
\sigma_{t}^{2}=\frac{\omega}{1-\beta}+\alpha \sum \beta^{j-1} r_{t-j}^{2}
$$

so that the $\operatorname{GARCH}(1,1)$ process implies that current volatility is an exponentially weighted moving average of past squared returns. Hence the $\operatorname{GARCH}(1,1)$ volatility measurement is seemingly, and perhaps surprisingly, very similar to RM volatility measurement. There are crucial differences, however.

First, GARCH parameters, and hence ultimately GARCH volatility, are estimated using rigorous statistical methods that facilitate probabilistic inference, in contrast to exponential smoothing in which the parameter is set in an ad hoc fashion. Typically we estimates the vector of GARCH parameters $\theta$ by maximizing the log likelihood function,

$$
\log L\left(\theta ; r_{w, T}, \ldots, r_{w, 1}\right) \propto-\sum_{t=1}^{T}\left[\log \sigma_{t}^{2}(\theta)-\sigma_{t}^{-2}(\theta) r_{w, t}^{2}\right]
$$

Note that the assumption of conditional normality underlying the (quasi) likelihood function in (8) is merely a matter of convenience, allowing for the calculation of consistent and asymptotic normal parameter estimates. The conditional return distribution will generally not be normal. The log-likelihood optimization in (9) can only be done numerically. However, GARCH models are parsimonious and specified directly in terms of univariate portfolio returns, so that only a single numerical optimization needs to be performed. This optimization can be performed in a matter of seconds on a standard desktop computer using standard MBA-ware, as discussed by 
Christoffersen (2003). ${ }^{5}$

Second, the covariance stationary $\operatorname{GARCH}(1,1)$ process has dynamics that eventually produce reversion in volatility to a constant long-run value, which enables interesting and realistic forecasts. This contrasts sharply with the RM exponential smoothing approach. As is well-known (e.g., Nerlove and Wage, 1964, Theil and Wage, 1964), exponential smoothing is optimal if and only if squared returns follow a "random walk plus noise" model (a "local level" model in the terminology of Harvey, 1989), in which case the minimum MSE forecast at any horizon is simply the current smoothed value. The historical records of volatilities of numerous assets (not to mention the fact that volatilities are bounded below by zero) suggest, however, that volatilities are unlikely to follow random walks, and hence that the flat forecast function associated with exponential smoothing is unrealistic and undesirable for volatility forecasting purposes.

Let us elaborate. We can rewrite the $\operatorname{GARCH}(1,1)$ model in (7) as

$$
\sigma_{t}^{2}=(1-\alpha-\beta) \sigma^{2}+\alpha r_{w, t-1}^{2}+\beta \sigma_{t-1}^{2},
$$

where $\sigma^{2} \equiv \omega /(1-\alpha-\beta)$ denotes the long-run, or unconditional daily variance. This representation shows that the GARCH forecast is constructed as an average of three elements. Equivalently we can also write the model as

$$
\sigma_{t}^{2}=\sigma^{2}+\alpha\left(r_{w, t-1}^{2}-\sigma^{2}\right)+\beta\left(\sigma_{t-1}^{2}-\sigma^{2}\right),
$$

which explicitly shows how the $\mathrm{GARCH}(1,1)$ model forecast by making adjustments to the current variance and the influence of the squared return around the long-run, or unconditional variance. Finally, we can also write

$$
\sigma_{t}^{2}=\sigma^{2}+(\alpha+\beta)\left(\sigma_{t-1}^{2}-\sigma^{2}\right)+\alpha \sigma_{t-1}^{2}\left(z_{t-1}^{2}-1\right),
$$

where the last term on the right-hand-side on average is equal to zero. Hence, this shows how the $\operatorname{GARCH}(1,1)$ forecasts by making adjustments around the long-run variance with variance persistence governed by $(\alpha+\beta)$ and the (contemporaneous) volatility-of-volatility linked to the level of volatility as well as the size of $\alpha$.

The mean-reverting property of GARCH volatility forecasts has important implications for the volatility term structure. To construct the volatility term structure corresponding to a $\operatorname{GARCH}(1,1)$ model, we need the k-day ahead variance forecast, which is

$$
\sigma_{t+k \mid t}^{2}=\sigma^{2}+(\alpha+\beta)^{k-1}\left(\sigma_{t+1}^{2}-\sigma^{2}\right) .
$$

${ }^{5}$ For further discussion of inference in GARCH models see also Andersen, Bollerslev, Christoffersen and Diebold (2005). 
Assuming that the daily returns are serially uncorrelated, the variance of the k-day cumulative returns, which we use to calculate the volatility term structure, is then

$$
\sigma_{t: t+k \mid t}^{2}=k \sigma^{2}+\left(\sigma_{t+1}^{2}-\sigma^{2}\right)\left(1-(\alpha+\beta)^{k}\right)(1-\alpha-\beta)^{-1} .
$$

Compare this mean-reverting expression with the RM forecast in (5). In particular, note that the speed of mean-reversion in the GARCH $(1,1)$ model is governed by $\alpha+\beta$. The mean-reverting line in Figure 3 above is calculated from (12), normalizing by $\mathrm{k}$ and taking the square root to display the graph in daily standard deviation units.

Third, the dynamics associated with the $\operatorname{GARCH}(1,1)$ model afford rich and intuitive interpretations, and they are readily generalized to even richer specifications. To take one important example, note that the dynamics may be enriched via higher-ordered specifications, such as GARCH(2,2). Indeed, Engle and Lee (1999) show that the GARCH(2,2) is of particular interest, because under certain parameter restrictions it implies a component structure obtained by allowing for time variation in the long-run variance in (10),

$$
\sigma_{t}^{2}=q_{t}+\alpha\left(r_{w, t-1}^{2}-q_{t}\right)+\beta\left(\sigma_{t-1}^{2}-q_{t}\right)
$$

with the long-run component, $\mathrm{q}_{\mathrm{t}}$, modeled as a separate autoregressive process,

$$
q_{t}=\omega+\rho q_{t-1}+\phi\left(r_{w, t-1}^{2}-\sigma_{t-1}^{2}\right)
$$

Many authors, including Gallant, Hsu and Tauchen (1999) and Alizadeh, Brandt and Diebold (2002) have found evidence of component structure in volatility, suitable generalizations of which can be shown to approximate long memory (e.g., Andersen and Bollerslev, 1997, and Barndorff-Nielsen and Shephard, 2001), which is routinely found in asset return volatilities (e.g., Bollerslev and Mikkelsen, 1999).

To take a second example of the extensibility of GARCH models, note that all models considered thus far imply symmetric response to positive vs. negative return shocks. However, equity markets, and particularly equity indexes, often seem to display a strong asymmetry, whereby a negative return boosts volatility by more than a positive return of the same absolute magnitude. The GARCH model is readily generalized to capture this effect. In particular, the asymmetric GJR-GARCH(1,1) model of Glosten, Jagannathan and Runkle (1993) is simply defined by

$$
\sigma_{t}^{2}=\omega+\alpha r_{w, t-1}^{2}+\gamma r_{w, t-1}^{2} \mathbf{1}\left(r_{w, t-1}<0\right)+\beta \sigma_{t-1}^{2}
$$

Asymmetric response in the conventional direction thus occurs when $\gamma>0$. $^{6}$

${ }^{6}$ Engle $(2001,2004)$ demonstrate empirically how allowing for asymmetries in the conditional variance can materially affect GARCH-based VaR calculations. 


\section{Asset Level Analysis: Modeling Asset Return Covariance Matrices}

The discussion above focused on the specification of dynamic volatility models for the aggregate portfolio return. These methods are well-suited for providing forecasts of portfolio level risk measures such as the aggregate VaR. However they are less well-suited for providing input into the active portfolio and risk management process. If, for example, the portfolio manager wants to know the optimal portfolio weights to minimize portfolio variance, then a multivariate model, which provides a forecast for the entire covariance matrix, is needed. ${ }^{7}$

Multivariate models are also better suited for calculating sensitivity risk measures to answer questions such as: "If I add an additional 1,000 shares of IBM to my portfolio, how much will my VaR increase?" Moreover, bank-wide VaR is made up of many desks with multiple traders on each desk, and any sub-portfolio analysis is not possible with the aggregate portfoliobased approach. ${ }^{8}$

In this section we therefore consider the specification of models for the full Ndimensional conditional distribution of asset returns. Generalizing the expression in (6), we write the multivariate model as

$$
R_{t}=\Omega_{t}^{1 / 2} Z_{t} \quad Z_{t} \sim \text { i.i.d. } \quad E\left(Z_{t}\right)=0 \quad \operatorname{Var}\left(Z_{t}\right)=I,
$$

where we have again set the mean to zero and where $I$ denotes the identity matrix. The $\mathrm{N} \times \mathrm{N}$ $\Omega_{t}^{1 / 2}$ matrix can be thought of as the square-root, or Cholesky decomposition, of the covariance matrix $\Omega_{t}$. This section will focus on specifying a dynamic model for this matrix, while section 5 will suggest methods for specifying the distribution of the innovation vector $Z_{\mathrm{t}}$.

Constructing positive semidefinite (psd) covariance matrix forecasts, which ensures that the portfolio variance is always non-negative, presents a key challenge below. The covariance matrix will have $1 / 2 \mathrm{~N}(\mathrm{~N}+1)$ distinct elements, but structure needs to be imposed to guarantee psd. The practical issues involved in estimating the parameters guarding the dynamics for the $1 / 2 \mathrm{~N}(\mathrm{~N}+1)$ elements are related and equally important. Although much of the academic literature focuses on relatively small multivariate examples, in this section we will confine attention to methods that are applicable even with $\mathrm{N}$ is (relatively) large.

\subsection{Covariance Matrices via Exponential Smoothing}

${ }^{7}$ Brandt, Santa-Clara and Valkanov (2004) provide an alternative and intriguing new approach for achieving dimension reduction, by explicitly parameterizing the portfolio weights as a function of directly observable state variables, thereby sidestepping the need to estimate the full covariance matrix; see also Pesaran and Zaffaroni (2004).

${ }^{8}$ See Manganelli (2004) for an interesting new low-dimensional approach to this problem. 
The natural analogue to the RM variance dynamics in (3) assumes that the covariance matrix dynamics are driven by the single parameter $\lambda$ for all variances and covariance in $\Omega_{t}$ :

$$
\Omega_{t}=\lambda \Omega_{t-1}+(1-\lambda) R_{t-1} R_{t-1}^{\prime}
$$

The covariance matrix recursion may again be initialized by setting $\Omega_{0}$ equal to the sample average coverage matrix.

The RM approach is clearly very restrictive, imposing the same degree of smoothness on all elements of the estimated covariance matrix. Moreover, covariance matrix forecasts generated by RM are in general suboptimal, for precisely the same reason as with the univariate $\mathrm{RM}$ variance forecasts discussed earlier. If the multivariate RM approach has costs, it also has benefits. In particular, the simple structure in (17) immediately guarantees that the estimated covariance matrices are psd, as the outer product of the return vector must be psd unless some assets are trivial linear combinations of others. Moreover, as long as the initial covariance matrix is psd (which will necessarily be the case when we set $\Omega_{0}$ equal to the sample average coverage matrix as suggested above, so long as the sample size $\mathrm{T}$ is larger than the number of assets $\mathrm{N}$ ), $\mathrm{RM}$ covariance matrix forecasts will also be psd, because a sum of positive semi-definite matrices is itself positive semi-definite.

\section{$\underline{3.2 \text { Covariance Matrices via Multivariate GARCH }}$}

Although easily implemented, the RM approach (17) may be much too restrictive in many cases. Hence we now consider multivariate GARCH models. The most general multivariate GARCH $(1,1)$ model is

$$
\operatorname{vech}\left(\Omega_{t}\right)=\operatorname{vech}(C)+B \operatorname{vech}\left(\Omega_{t-1}\right)+A \operatorname{vech}\left(R_{t-1} R_{t-1}^{\prime}\right)
$$

where the vech ("vector half") operator converts the unique upper triangular elements of a symmetric matrix into a $1 / 2 \mathrm{~N}(\mathrm{~N}+1) \times 1$ column vector, and $A$ and $B$ are $1 / 2 \mathrm{~N}(\mathrm{~N}+1) \times 1 / 2 \mathrm{~N}(\mathrm{~N}+1)$ matrices. Notice that in this general specification, each element of $\Omega_{\mathrm{t}-1}$ may potentially affect each element of $\Omega_{t}$, and similarly for the outer product of past returns, producing a serious "curse-of-dimensionality" problem. In its most general form the GARCH $(1,1)$ model $(18)$ has a total of $1 / 2 \mathrm{~N}^{4}+\mathrm{N}^{3}+\mathrm{N}^{2}+1 / 2 \mathrm{~N}=\mathrm{O}\left(\mathrm{N}^{4}\right)$ parameters. Hence, for example, for $\mathrm{N}=100$ the model has 51,010,050 parameters! Estimating this many free parameters is obviously infeasible. Note also that without specifying more structure on the model there is no guarantee of positive definiteness of the fitted or forecasted covariance matrices.

The dimensionality problem can be alleviated somewhat by replacing the constant term via "variance targeting" as suggested by Engle and Mezrich (1996):

$$
\operatorname{vech}(C)=(I-A-B) \operatorname{vech}\left(\frac{1}{T} \sum_{t=1}^{T} R_{t} R_{t}^{\prime}\right) \text {. }
$$


This is also very useful from a forecasting perspective, as small perturbations in A and B sometimes result in large changes in the implied unconditional variance to which the long-run forecasts converge. However, there are still too many parameters to be estimated simultaneously in $\mathrm{A}$ and $\mathrm{B}$ in the general multivariate model when $\mathrm{N}$ is large.

More severe (and hence less palatable) restrictions may be imposed to achieve additional parsimony, as for example with the "diagonal GARCH" pameterization proposed by Bollerslev, Engle and Wooldridge (1988). In a diagonal GARCH model, the matrices A and B have zeros in all off-diagonal elements, which in turn implies that each element of the covariance matrix follows a simple dynamic with univariate flavor: conditional variances depend only on own lags and own lagged squared returns, and conditional covariances depend only on own lags and own lagged cross products of returns. Even the diagonal GARCH framework, however, results in $\mathrm{O}\left(\mathrm{N}^{2}\right)$ parameters to be jointly estimated, which is computationally infeasible in systems of medium and large size.

One approach is to move to the most draconian version of the diagonal GARCH model, in which the matrices $B$ and $A$ are simply scalar matrices. Specifically,

$$
\Omega_{t}=C+\beta \Omega_{t-1}+\alpha\left(R_{t-1} R_{t-1}^{\prime}\right)
$$

where the value of each diagonal element of $B$ is $\beta$, and each diagonal element of $A$ is $\alpha$. Rearrangement yields

$$
\Omega_{t}=\Omega+\beta\left(\Omega_{t-1}-\Omega\right)+\alpha\left(R_{t-1} R_{t-1}^{\prime}-\Omega\right),
$$

which is closely related to the multivariate RM approach, with the important difference that it introduces, a non-degenerate long-run covariance matrix $\Omega$ to which $\Omega_{t}$ reverts (provided that $\alpha+\beta<1$ ). Notice also though that all variance and covariances are assumed to have the same speed of mean reversion, because of common $\alpha$ and $\beta$ parameters, which may be overly restrictive.

\subsection{Dimensionality Reduction I: Covariance Matrices via Flex-GARCH}

Ledoit, Santa-Clara and Wolf (2003) suggest an attractive "Flex-GARCH" method for reducing the computational burden in the estimation of the diagonal GARCH model without moving to the scalar version. Intuitively, Flex-GARCH decentralizes the estimation procedure by estimating $\mathrm{N}(\mathrm{N}+1) / 2$ bivariate GARCH models with certain parameter constraints, and then "pasting" them together to form the matrices A, B, and C in (18). Specific transformations of the parameter matrices from the bivariate models ensure that the resulting conditional covariance matrix forecast is psd. Flex-GARCH appears to be a viable modeling approach when $\mathrm{N}$ is larger than say five, where estimation of the general diagonal GARCH model becomes intractable. However, when $\mathrm{N}$ is of the order of thirty and above, which is often the case in practical risk management applications, it becomes cumbersome to estimate $\mathrm{N}(\mathrm{N}+1) / 2$ bivariate models, and alternative dimensionality reduction methods are necessary. One such method is the dynamic 
conditional correlation framework, to which we now turn.

\subsection{Dimensionality Reduction II: Covariance Matrices via Dynamic Conditional Correlation}

Recall the simple but useful decomposition of the covariance matrix into the correlation matrix pre- and post-multiplied by the diagonal standard deviation matrix,

$$
\Omega_{t} \equiv D_{t} \Gamma_{t} D_{t}
$$

Bollerslev (1990) uses this decomposition, along with an assumption of constant conditional correlations $\left(\Gamma_{\mathrm{t}}=\Gamma\right)$ to develop his Constant Conditional Correlation (CCC) GARCH model. The assumption of constant conditional correlation, however, is arguably too restrictive over long time periods.

Engle (2002) generalizes Bollerslev's (1990) CCC model to obtain a Dynamic Conditional Correlation (DCC) model. Crucially, he also provides a decentralized estimation procedure. First, one fits to each asset return an appropriate univariate GARCH model (the models can differ from asset to asset) and then standardizes the returns by the estimated GARCH conditional standard deviations. Then one uses the standardized return vector, say $e_{t} \equiv R_{t} \hat{D}_{t}^{-1}$, to model the correlation dynamics. For instance, a simple scalar diagonal $\operatorname{GARCH}(1,1)$ correlation dynamic would be

$$
Q_{t}=C+\beta Q_{t-1}+\alpha\left(e_{t-1} e_{t-1}^{\prime}\right)
$$

with the individual correlations in the $\Gamma_{\mathrm{t}}$ matrix defined by the corresponding normalized elements of $\mathrm{Q}_{\mathrm{t}}$,

$$
\rho_{i, j, t}=q_{i, j, t} /\left(\sqrt{q_{i, i, t}} \sqrt{q_{j, j, t}}\right) \text {. }
$$

The normalization in (24) ensures that all correlation forecasts fall in the $[-1 ; 1]$ interval, while the simple scalar structure for the dynamics of $Q_{t}$ in (23) ensures that $\Gamma_{t}$ is psd.

If $\mathrm{C}$ is pre-estimated by correlation targeting, as discussed earlier, only two parameters need to be estimated in (23). Estimating variance dynamics asset-by-asset and then assuming a simple structure for the correlation dynamics thus ensures that the DCC model can be implemented in large systems: $\mathrm{N}+1$ numerical optimizations must be performed, but each involves only a few parameters, regardless of the size of $\mathrm{N}$.

Although the DCC model offers a promising framework for exploring correlation dynamics in large systems, the simple dynamic structure in (23) may be too restrictive for many applications. For example, volatility and correlation responses may be asymmetric in the signs of 
past shocks. ${ }^{9}$ Researchers are therefore currently working to extend the DCC model to more general dynamic correlation specifications. Relevant work includes Franses and Hafner (2003), Pelletier (2004), and Cappiello, Engle, and Sheppard (2004).

To convey a feel for the importance of allowing for time-varying conditional correlation, we show in Figure 4 the bond return correlation between Germany and Japan estimated using a DCC model allowing for asymmetric correlation responses to positive versus negative returns, reproduced from Cappiello, Engle, and Sheppard (2004). The conditional correlation clearly varies a great deal. Note in particular the dramatic change in the conditional correlation around the time of the Euro's introduction in 1999. Such large movements in conditional correlation are not rare, and they underscore the desirability of allowing for different dynamics in volatility versus correlation. ${ }^{10}$

\section{Exploiting High-Frequency Return Data for Improved Covariance Matrix Measurement}

Thus far our discussion has implicitly focused on models tailored to capturing the dynamics in returns by relying only on daily return information. For many assets, however, highfrequency price data are available and should be useful for the estimation of asset return variances and covariances. Here we review recent work in this area and speculate on its usefulness for constructing large-scale models of market risk.

\subsection{Realized Variances}

Following Andersen, Bollerslev, Diebold and Labys (2003) (henceforth ABDL), define the realized variance $(\mathrm{RV})$ on day $\mathrm{t}$ using returns constructed at the $\Delta$ intra-day frequency as

$$
\sigma_{t, \Delta}^{2} \equiv \sum_{j=1}^{1 / \Delta} r_{t-1+j \Delta, \Delta}^{2},
$$

where $1 / \Delta$ is, for example, 48 for 30 -minute returns in 24 -hour markets. Theoretically, letting $\Delta$ go to zero, which implies sampling continuously, we approach the true integrated volatility of the underlying continuous time process on day . $^{11}$

${ }^{9}$ A related example is the often-found positive relationship between volatility changes and correlation changes. If present but ignored, this effect can have serious consequences for portfolio hedging effectiveness.

10 As another example, cross-market stock-bond return correlations are often found to be close to zero or slightly positive during bad economic times (recessions), but negative in good economic times (expansions); see, e.g., the discussion in Andersen, Bollerslev, Diebold and Vega (2004).

${ }^{11}$ For a full treatment, see Andersen, Bollerslev and Diebold (2004). 
In practice, market microstructure noise will affect the RV estimate when $\Delta$ gets too small. Prices sampled at 15-30 minute intervals, depending on the market, are therefore often used. Notice also that, in markets that are not open 24 hours per day, the potential jump from the closing price on day $\mathrm{t}-1$ to the opening price on day $\mathrm{t}$ must be accounted for. This can be done using the method in Hansen and Lunde (2004). As is the case for the daily GARCH models considered above, corrections may also have to be made for the fact that days following weekends and holidays tend to have higher than average volatility.

Although the daily realized variance is just an estimate of the underlying integrated variance and likely measured with some error, it presents an intriguing opportunity: it is potentially highly accurate, and indeed accurate enough such that we might take the realized daily variance as an observation of the true daily variance, modeling and forecasting it using standard ARMA time series tools. Allowing for certain kinds of measurement error can also easily be done in this framework. The upshot is that if the fundamental frequency of interest is daily, then using sufficiently high-quality intra-day price data enables the risk manager to treat volatility as essentially observed. This is vastly different from the GARCH style models discussed above, in which the daily variance is constructed recursively from past daily returns.

As an example of the direct modeling of realized volatility, one can specify a simple firstorder autoregressive model for the log realized volatility,

$$
\log \left(\sigma_{t, \Delta}\right) \equiv c+\beta \log \left(\sigma_{t-1, \Delta}\right)+v_{t},
$$

which can be estimated using simple OLS. The log specification guarantees positivity of forecasted volatilities and induces (approximate) normality, as demonstrated empirically in Andersen, Bollerslev, Diebold and Labys (2000, 2001). ABDL show the superior forecasting properties of RV-based forecasts compared with GARCH forecasts. Rather than relying on a simple short-memory ARMA model as in (26), they specify a fractionally integrated model to better account for the apparent long-memory routinely found in volatility dynamics.

Along these lines, Figure 5 shows clear evidence of long-memory in foreign exchange RVs as evidenced by the sample autocorrelation function for lags of 1 through 100 days. We first construct the daily RVs from 30-minute FX returns and then calculate the corresponding daily sample autocorrelations of the RVs. Note that the RV autocorrelations are significantly positive for all 100 lags when compared with the conventional 95-percent Bartlett confidence bands.

The RV forecasts may also be integrated into the standard GARCH modeling framework, as explored in Engle and Gallo (2004). ${ }^{12}$ Similarly, rather than relying on GARCH variance models to standardize returns in the first step of the DCC model, RVs can be used instead. Doing

${ }^{12}$ Intriguing new procedures for combining high-frequency data and RV type measures with lower frequency daily returns in volatility forecasting models have also recently been developed by Ghysels, Santa-Clara and Valkanov (2005). 
so would result in a more accurate standardization and would require only a single numerical optimization step - estimation of correlation dynamics - thereby rendering the computational burden in DCC nearly negligible.

We next discuss how realized variances and their natural multivariate counterparts, realized covariances, can be used in a more systematic fashion in risk management.

\section{$\underline{4.2}$ Realized Covariances}

Generalizing the realized variance idea to the multivariate case, we can define the daily realized covariance matrix as

$$
\Omega_{t, \Delta} \equiv \sum_{j=1}^{1 / \Delta} R_{t-1+j \Delta, \Delta} R_{t-1+j \Delta, \Delta}^{\prime} .
$$

The upshot again is that variances and covariances no longer have to be extracted from a nonlinear model estimated via treacherous maximum likelihood procedures, as was the case for the GARCH models above. Using intra-day price observations, we essentially observe the daily covariances and can model them as if they were observed. ABDL show that, as long as the asset returns are linearly independent and $\mathrm{N}<1 / \Delta$, the realized covariance matrix will be positive definite. However $1 / \Delta$ is, for example, 48 , and so in large portfolios the condition is likely to be violated. We return to this important issue at the end of this section.

Microstructure noise may plague realized covariances, just as it may plague realized variances. Non-synchronous trading, however, creates additional complications in the multivariate case. These are similar, but potentially more severe, than the non-synchronous trading issues that arise in the estimation of say, monthly covariances and CAPM betas with nonsynchronous daily data. A possible fix involves inclusion of additional lead and lag terms in the realized covariance measure (27), along the lines of the Scholes and Williams (1977) beta correction technique. Work on this is still in its infancy, and we will not discuss it any further here, but an important recent contribution is Martens (2004).

We now consider various strategies for modeling and forecasting realized covariances, treating them as directly observable vector time series. These all are quite speculative, as little work has been done to date in terms of actually assessing the economic value of using realized covariances for practical risk measurement and management problems. ${ }^{13}$

Paralleling the tradition of the scalar diagonal GARCH model, directly suggests the following model

${ }^{13}$ One notable exception is the work of Fleming, Kirby, Oestdiek (2003), which suggests dramatic improvements vis-a-vis the RM and multivariate GARCH frameworks for standard mean-variance efficient asset allocation problems. 


$$
\operatorname{vech}\left(\Omega_{t, \Delta}\right)=\operatorname{vech}(C)+\beta \operatorname{vech}\left(\Omega_{t-1, \Delta}\right)+v_{t},
$$

which requires nothing but simple OLS to implement, while guaranteeing positive definiteness of the corresponding covariance matrix forecasts for any positive definite matrix $\mathrm{C}$ and positive values of $\beta$. This does again however impose a common mean-reversion parameter across variances and covariances, which may be overly restrictive. Realized covariance versions of the non-scalar diagonal GARCH model could be developed in a similar manner, keeping in mind the restrictions required for positive definiteness.

Positive definiteness may also be imposed by modeling the Cholesky decomposition of the realized covariance matrix rather than the matrix itself, as suggested by ABDL. We have

$$
\Omega_{t, \Delta} \equiv P_{t, \Delta} P_{t, \Delta}^{\prime},
$$

where $\mathrm{P}_{t, \Delta}$ is a unique lower triangular matrix. The data vector is then vech $\left(\mathrm{P}_{t, \Delta}\right)$, and we substitute the forecast of vech $\left(\mathrm{P}_{\mathrm{t}+\mathrm{k}, \Delta}\right)$ back into (29) to construct a forecast of $\Omega_{t+k, \Delta}$.

Alternatively, in the tradition of Ledoit and Wolf (2003), one may encourage positive definiteness of high-dimensional realized covariance matrices by shrinking toward the covariance matrix implied by a single-factor structure, in which the optimal shrinkage parameter is estimated directly from the data.

We can also use a DCC-type framework for realized correlation modeling. In parallel to (21) we write

$$
\Omega_{t, \Delta} \equiv D_{t, \Delta} \Gamma_{t, \Delta} D_{t, \Delta},
$$

where the typical element in the diagonal matrix $D_{t, \Delta}$ is the realized standard deviation, and the typical element in $\Gamma_{t, \Delta}$ is constructed from the elements in $\Omega_{t, \Delta}$ as

$$
\rho_{i, j, t, \Delta} \equiv \sigma_{i, j, t, \Delta} /\left(\sigma_{i, i, t, \Delta} \sigma_{j, j, t, \Delta}\right) .
$$

Following the DCC idea, we model the standard deviations asset-by-asset in the first step, and the correlations in a second step. Keeping a simple structure as in (23), we have

$$
\operatorname{vech}\left(Q_{t, \Delta}\right)=\operatorname{vech}(C)+\beta \operatorname{vech}\left(Q_{t-1, \Delta}\right)+v_{t},
$$

where simple OLS again is all that is required for estimation. Once again, a normalization is needed to ensure that the correlation forecasts fall in the $[-1 ; 1]$ interval. Specifically,

$$
\hat{\rho}_{i, j, t, \Delta}=\hat{q}_{i, j, t, \Delta} /\left(\sqrt{\hat{q}_{i, i, t, \Delta}} \sqrt{\hat{q}_{j, j, t, \Delta}}\right) .
$$

The advantages of this approach are twofold: first, high-frequency information is used to obtain more precise forecasts of variances and correlations. Second, numerical optimization is not 
needed at all. Long-memory dynamics or regime-switching could, of course, be incorporated as well.

Although there appear to be several avenues for exploiting intra-day price information in daily risk management, two key problems remain. First, many assets in typical portfolios are not liquid enough for intraday information to be available and useful. Second, even in highly-liquid environments, when $\mathrm{N}$ is very large the positive definiteness problem remains. We now explore a potential solution to these problems.

\subsection{Dimensionality Reduction III: (Realized) Covariance Matrices via Mapping to Liquid Base Assets}

Multivariate market risk management systems for portfolios of thousands of assets often work from a set of, say, 30 observed base assets believed to be key drivers of risk. The choice of these, of course, depend on the portfolio at hand but can, for example, consist of equity market indices, FX rates, benchmark interest rates, and so on, which are believed to capture the main sources of uncertainty in the portfolio. The assumptions made on the multivariate distribution of base assets are naturally of crucial importance for the accuracy of the risk management system.

Note that base assets typically correspond to the most liquid assets in the market. The upshot here is that we can credibly rely on realized volatility and covariances in this case. Using the result from ABDL, a base asset system of dimension $\mathrm{N}_{\mathrm{F}}<1 / \Delta$ will ensure that the realized covariance matrix is psd and therefore useful for forecasting.

The mapping from base assets to the full set of assets is discussed in Jorion (2000). In particular, the factor model is naturally expressed as ${ }^{14}$

$$
R_{t}=\mathrm{B} R_{F, t}+v_{t}
$$

where $v_{t}$ denotes the idiosyncratic risk. The factor loadings in the $\mathrm{N} \times \mathrm{N}_{\mathrm{F}}$ matrix $\mathrm{B}$ may be obtained from regression (if data exists), or via pricing model sensitivities (if a pricing model exists). Otherwise the loadings may be determined by ad hoc considerations such as matching a security without a well-defined factor loading to another similar security which has a welldefined factor loading.

We now need a multivariate model for the $\mathrm{N}_{\mathrm{F}}$ base assets. However, assuming that

$$
R_{F, t}=\Omega_{F, t}^{1 / 2} Z_{F, t} \quad Z_{F, t} \sim \text { i.i.d. } \quad E\left(Z_{F, t}\right)=0 \quad \operatorname{Var}\left(Z_{F, t}\right)=I,
$$

we can use the modeling strategies discussed above to construct the $\mathrm{N}_{\mathrm{F}} \times \mathrm{N}_{\mathrm{F}}$ realized factor

\footnotetext{
${ }^{14}$ Diebold and Nerlove (1989) construct a multivariate ARCH factor model in which the latent time-varying volatility factors can be viewed as the base assets.
} 
covariance matrix $\Omega_{F, t}$ and the resulting systematic covariance matrix measurements and forecast.

\section{Modeling Entire Conditional Return Distributions}

Proper portfolio and risk management requires knowing the entire multivariate distribution of asset or base asset returns, not just the second moments. Conventional risk measures such as VaR and expected shortfall, however, capture only limited aspects of the distribution.

In this section we therefore explore various approaches to complete the model. Notice that above we deliberately left the distributional assumption on the standardized returns unspecified. We simply assumed that the standardized returns were i.i.d. We will keep the assumption of i.i.d. standardized returns below and focus on ways to estimate the constant conditional density. This is, of course, with some loss of generality as dynamics in moments beyond second-order could be operative. The empirical evidence for such higher-ordered conditional moment dynamics is, however, much less conclusive at this stage.

The evidence that daily standardized returns are not normally distributed is, however, quite conclusive. Although GARCH and other dynamic volatility models do remove some of the non-normality in the unconditional returns, conditional returns still exhibit non-normal features. Interestingly, these features vary systematically from market to market. For example, mature FX market returns are generally strongly conditionally kurtotic, but approximately symmetric. Meanwhile, most aggregate index equity returns appear to be both conditionally skewed and fat tailed.

As an example of the latter, we show in Figure 6 the daily QQ plot for S\&P500 returns from January 2, 1990 to December 31, 2002, standardized using the (constant) average daily volatility across the sample. That is, we plot quantiles of standardized returns against quantiles of the standard normal distribution. Clearly the daily returns are not unconditionally normally distributed. Consider now Figure 7 in which the daily returns are instead standardized by the time-varying volatilities from an asymmetric GJR GARCH(1,1) model. The QQ plot in Figure 7 makes clear that although the GARCH innovations conform more closely to the normal distribution than do the raw returns, the left tail of the S\&P500 returns conforms much less well to the normal distribution than does the right tail: there are more large innovations than one would expect under normality.

As the VaR itself is a quantile, the QQ plot also gives an assessment of the accuracy of the normal-GARCH VaR for different coverage rates. Figure 7 suggests that a normal-GARCH VaR would work well for any coverage rate for a portfolio which is short the S\&P500. It may also work well for a long portfolio but only if the coverage rate is relatively large, say in excess of $5 \%$.

Consider now instead the distribution of returns standardized by realized volatility. In 
contrast to the poor fit in the left tail evident in Figure 7, the distribution in Figure 8 is strikingly close to normal, as first noticed by Zhou (1996) and Andersen, Bollerslev, Diebold and Labys (2000). Figures 7 and 8 rely on the same series of daily S\&P500 returns but simply use two different volatility measures to standardize the raw returns. The conditional non-normality of daily returns has been a key stylized fact in market risk management. Finding a volatility measure which can generate standardized returns that are close to normal is therefore surprising and noteworthy.

Figure 8 and the frequently-found lognormality of realized volatility itself suggest that a good approximation to the distribution of returns may be obtained using a normal / log-normal mixture model. In this model, the standardized return is normal and the distribution of realized volatility at time $t$ conditional on time $\mathrm{t}-1$ information is log-normal. This idea is explored empirically in ABDL, who find that a log-normal / normal mixture VaR model performs very well in an application to foreign exchange returns.

The recent empirical results in Andersen, Bollerslev and Diebold (2003) suggest that even better results may be obtained by separately measuring and modeling the part of the realized volatility attributable to "jumps" in the price process through so-called realized bipower variation measures, as formally developed by Barndorff-Nielsen and Shephard (2004). These results have great potential for application in financial risk management, and their practical implications are topics of current research.

Although realized volatility measures may be available for highly liquid assets, it is often not possible to construct realized volatility based portfolio risk measures. We therefore now survey some of the more conventional methods first for univariate and then for multivariate models.

\subsection{Portfolio Level: Univariate Analytic Methods}

Although the normal assumption works well in certain cases, we want to consider alternatives that allow for fat tails and asymmetry in the conditional distribution, as depicted in Figure 7. In the case of $\mathrm{VaR}$ we are looking for ways to calculate the cut-off $z_{p}^{-1}$ in

$$
\operatorname{VaR}_{T+1 \mid T}^{p} \equiv \sigma_{T+1} z_{p}^{-1}
$$

Perhaps the most obvious approach is simply to look for a parametric distribution more flexible than the normal while still tightly parameterized. One such example is the (standardized) Student's t distribution suggested by Bollerslev (1987), which relies on only one additional parameter in generating symmetric fat tails. Recently, generalizations of the Student's $t$ which allow for asymmetry have also been suggested, as in Fernandez and Steel (1998) and Hansen (1994).

Rather than assuming a particular parametric density, one can approximate the quantiles of non-normal distributions via Cornish-Fisher approximations. Baillie and Bollerslev (1992) first advocated this approach in the context of GARCH modeling and forecasting. The only 
inputs needed are the sample estimates of skewness and kurtosis of the standardized returns. Extreme value theory provides another approximation alternative, in which the tail(s) of the conditional distribution is estimated using only the extreme observations, as suggested in Diebold, Schuermann, and Stroughair (1998) and McNeil and Frey (2000).

A common problem with most GARCH models, regardless of the innovation distribution, is that the conditional distribution of returns is not preserved under temporal aggregation. Hence even if the standardized daily returns from a $\operatorname{GARCH}(1,1)$ model were normal, the implied weekly returns will not be. This in turn implies that the term structure of VaR or expected shortfall needs to be calculated via Monte Carlo simulation, as in, e.g., Guidolin and Timmermann (2004). But Monte Carlo simulation requires a properly specified probability distribution which would rule out the Cornish-Fisher and extreme-value-theory approximations.

Heston and Nandi (2000) suggest a specific affine GARCH-normal model, which may work well for certain portfolios, and which combined with the methods of Albanese, Jackson and Wiberg (2004) allows for relatively easy calculation of the term structure of VaRs. In general, however, simulation methods are needed, and we now discuss a viable approach which combines a parametric volatility model with a data-driven conditional distribution.

\subsection{Portfolio Level: Univariate Simulation Methods}

Bootstrapping, or Filtered Historical Simulation (FHS), assumes a parametric model for the second moment dynamics but bootstraps from standardized returns to construct the distribution. At the portfolio level this is easy to do. Calculate the standardized pseudo portfolio returns as

$$
\hat{z}_{w, t}=r_{w, t} / \hat{\sigma}_{t}, \quad \text { for } t=1,2, \ldots, T,
$$

using one of the variance models from section 2. For the one-day-ahead VaR, we then simply use the order statistic for the standardized returns combined with the volatility forecast to construct,

$$
F H S-V a R_{T+1}^{p} \equiv \sigma_{T+1} \hat{z}_{w}((T+1) p) .
$$

Multi-day VaR requires simulating paths from the volatility model using the standardized returns sampled with replacement as innovations. This approach has been suggested by Diebold, Schuermann and Stroughair (1998), Hull and White (1998) and Barone-Adesi, Bourgoin and Giannopoulos (1998), who coined the term FHS. Pritsker (2001) also provides evidence on its effectiveness.

\subsection{Asset Level: Multivariate Analytic Methods}

Just as a fully specified univariate distribution is needed for complete risk measurement, 
so too is a fully specified multivariate distribution needed for proper portfolio and risk management. This in turn requires us to make an assumption about the multivariate (but constant) distribution of $Z_{t}$ in (16).

The results of Andersen, Bollerslev, Diebold and Labys (2000) suggest that, at least in the FX market, the multivariate distribution of returns standardized by the realized covariance matrix is again closely approximated by a normal distribution. So long as the realized volatilities are available, a multivariate version of the log-normal mixture model discussed in connection with Figure 8 above could therefore be developed.

As noted earlier, however, construction and use of realized covariance matrices may be problematic in situations when liquidity is not high, in which case traditional parametric models may be used. As in the univariate case, however, the multivariate normal distribution coupled with multivariate standardization using covariance matrices estimated from traditional parametric models, although obviously convenient, does not generally provide an accurate picture of tail risk. $^{15}$

A few analytic alternatives to the multivariate normal paradigm do exist, such as the multivariate Student's t distribution first considered by Harvey, Ruiz and Sentana (1992), along with the more recent related work by Glasserman, Heidelberger, and Shahbuddin (2002). Recently much attention has also been focused on the construction of multivariate densities from the marginal densities via copulas, as in Jondeau and Rockinger (2004) and Patton (2002), although the viability of the methods in very high-dimensional systems remains to be established.

Multivariate extreme value theory offers a tool for exploring cross-asset tail dependencies, which are not captured by standard correlation measures. For example, Longin and Solnik (2001) define and compute extreme correlations between monthly U.S. index returns and a number of foreign country indexes. In the case of the bivariate normal distribution, correlations between extremes taper off to zero as the thresholds defining the extremes get larger in absolute value. The actual equity data, however, behave quite differently. The correlation between negative extremes is much larger than the normal distribution would suggest. ${ }^{16}$ Such strong correlation between negative extremes is clearly a key risk management concern. Poon, Rockinger and Tawn (2004) explore the portfolio risk management implications of extremal dependencies, while Hartmann, Straetmans and de Vries (2004) consider their effect on banking system stability. Once again, however, it is not yet clear whether such methods will be operational in large-dimensional systems.

${ }^{15}$ In the multivariate case the normal distribution is even more tempting to use, because it implies that the aggregate portfolio distribution itself is also normally distributed.

${ }^{16}$ In contrast, and interestingly, the correlations of positive extremes appear to approach zero in accordance with the normal distribution. 
Issues of scalability, as well as cross-sectional and temporal aggregation problems in parametric approaches, thus once again lead us to consider simulation based solutions.

\subsection{Asset Level: Multivariate Simulation Methods}

In the general multivariate case, we can in principle use FHS with dynamic correlations, but a multivariate standardization is needed. Using the Cholesky decomposition, we first create vectors of standardized returns from (16). We write the standardized returns from an estimated multivariate dynamic covariance matrix as

$$
\hat{Z}_{t}=\hat{\Omega}_{t}^{-1 / 2} R_{t} \text { for } t=1,2, \ldots, T,
$$

where we calculate $\hat{\Omega}_{t}^{-1 / 2}$ from the Cholesky decomposition of the inverse covariance matrix $\hat{\Omega}_{t}^{-1}$. Now, resampling with replacement vector-wise from the standardized returns will ensure that the marginal distributions as wells as particular features of the multivariate distribution, as for example, the cross-sectional dependencies suggested by Longin and Solnik (2001), will be preserved in the simulated data.

The dimensionality of the system in (39) may render the necessary multivariate standardization practically infeasible. However, the same FHS approach can be applied with the base asset setup in (35), resampling from the factor innovations calculated as

$$
\hat{Z}_{F, t}=\hat{\Omega}_{F, t}^{-1 / 2} R_{F, t} \text { for } t=1,2, \ldots, T \text {, }
$$

where we again use the Cholesky decomposition to build up the distribution of the factor returns. From (34) we can then construct the corresponding idiosyncratic asset innovations as,

$$
\hat{v}_{t}=R_{t}-\hat{\mathrm{B}} R_{F, t} \text { for } t=1,2, \ldots, T \text {, }
$$

in turn resampling from $\hat{Z}_{t}$ and $\hat{v}_{t}$ to build up the required distribution of the individual asset returns in the base asset model.

Alternatively, if one is willing to assume constant conditional correlations, then the standardization can simply be done on an individual asset-by-asset basis using the univariate GARCH volatilities. Resampling vector-wise from the standardized returns will preserve the cross-sectional dependencies in the historical data.

\section{Summary and Directions for Future Research}

We have attempted to demonstrate the power and potential of dynamic financial econometric methods for practical financial risk management, surveying the large literature on high-frequency volatility measurement and modeling, interpreting and unifying the most important and intriguing results for practical risk management. The paper complements the more general and technical (and less financial) survey of volatility and covariance forecasting in 
Andersen, Bollerslev, Christoffersen and Diebold (2005).

\section{$\underline{6.1 \text { Summary }}$}

Our key points are as follows:

- Standard "model-free" methods, such as historical simulation, rely on false assumptions of independent returns. Reliable risk measurement requires a conditional density model that allows for time-varying volatility.

- For the purpose of risk measurement, specifying a univariate density model directly on the portfolio return is likely to be most accurate. RiskMetrics offers one possible approach, but the temporal aggregation properties-including the volatility term structure-of RiskMetrics appear to be counter-factual.

- GARCH volatility models offer a convenient and parsimonious framework for modeling key dynamic features of returns, including volatility mean-reversion, long-memory, and asymmetries.

- Although risk measurement can be done from a univariate model for a given set of portfolio weights, risk management requires a fully specified multivariate density model. Unfortunately, standard multivariate GARCH models are too heavily parameterized to be useful in realistic large-scale problems.

- Recent advances in multivariate GARCH modeling are likely to be useful for medium-scale models, but very large scale modeling requires decoupling variance and correlation dynamics, as in the dynamic conditional correlation model.

- Volatility measures based on high-frequency return data hold great promise for practical risk management. Realized volatility and correlation measures give more accurate forecasts of future realizations than their conventional competitors. Because high-frequency information is only available for highly liquid assets, we suggest a base-asset factor approach.

- Risk management requires fully-specified conditional density models, not just conditional covariance models. Resampling returns standardized by the conditional covariance matrix presents an attractive strategy for accommodating conditionally non-normal returns.

- The near log-normality of realized volatility, together with the near-normality of returns standardized by realized volatility, holds promise for relatively simple-to-implement lognormal / normal mixture models in financial risk management.

Several important topics have thus far been omitted from this survey. We conclude by 
outlining two key areas for future research, namely option valuation and the treatment of microstructure noise.

\section{$\underline{6.2 \text { Future Research I: Option Valuation }}$}

The seminal Black-Scholes-Merton (BSM) model has produced indispensable insight into the valuation of derivative securities. However, many empirical studies have shown that the assumptions of constant volatility and normal returns cause the BSM model systematically to misprice options. Hence we have developed increasingly sophisticated models to deal with the shortcomings of the BSM model. Nevertheless, practitioners often rely on curves fitted to the implied BSM volatility surface to compute option prices. Thus, there appears to be a disconnect between the academic and practitioner camps in this area.

One reason for the disconnect is clearly that the more sophisticated academic models involve an increasing number of unobserved parameters and volatility factors, rendering them much harder to implement than the simple BSM model. However, recent advances in the estimation of more complicate and empirically realistic continuous time option pricing models (e.g. Chernov and Ghysels, 2000, Eraker, 2004, and Pan, 2002) may help reduce that problem.

Another way to close the apparent gap is to develop potentially more tractable discretetime analogues of the continuous time models. Heston and Nandi (2000) thus define a discrete time GARCH version of Heston's (1993) stochastic volatility model, while Christoffersen, Heston and Jacobs (2004) extend the model to allow for conditionally non-normal returns.

We have already discussed the need to model the long-memory features of return volatility dynamics. Getting the volatility dynamics right is clearly key for option valuation. One way to approximate long memory is via component models. Bates (2000) studies continuous time volatility component models in derivative pricing contexts, and Christoffersen, Jacobs and Wang (2004) study discrete time analogues.

\section{$\underline{6.3 \text { Future Research II: Microstructure Noise }}$}

The potential usefulness of intra-day returns for daily risk management has been a key theme in our discussion so far. The realized volatility literature is still quite new, and many important practical considerations are still being explored. The influence of market microstructure noise on realized volatility is one such consideration.

The theoretical result giving integrated volatility as the limit of realized volatility when the sampling frequency goes to infinity is derived under the assumption of no market microstructure noise. When allowing for market microstructure noise, the simple limit result no longer holds, and sampling infinitely often - or in practice, "as often as possible" - will bias the measures. Bandi and Russel (2004a, 2004b) derive optimal finite sampling frequencies for given specifications of market microstructure noise. But the suggestion to sample "less often than possible," thus discarding information, may be inefficient, and Ait-Sahalia, Mykland and Zhang 
(2004) suggest adjustments which in principle allow for the use of all available price information rather than sampling at certain prespecified frequencies. 


\section{References}

Ait-Sahalia, Y., P. Mykland, and L. Zhang, 2004, How Often to Sample a Continuous-Time Process in the Presence of Market Microstructure Noise, Review of Financial Studies, forthcoming.

Albanese, C., K. Jackson, and P. Wiberg, 2004, A New Fourier Transform Algorithm for Value-at-Risk, Quantitative Finance, 4, 328-338.

Alizadeh, S., M. Brandt, and F. Diebold, 2002, Range-Based Estimation of Stochastic Volatility Models, Journal of Finance, 57, 1047-1091.

Andersen, T. and T. Bollerslev, 1997, Heterogeneous Information Arrivals and Return Volatility Dynamics: Uncovering the Long Run in High Frequency Returns, Journal of Finance, 52, 975-1005.

Andersen, T.G., T. Bollerslev, P. Christoffersen and F.X. Diebold, 2005, Volatility Forecasting, in G. Elliott, C. Granger and A. Timmermann (eds.), Handbook of Economic Forecasting. Amsterdam: North-Holland, forthcoming

Andersen, T.G., T. Bollerslev, and F.X. Diebold, 2003, Some Like it Smooth, and Some Like it Rough: Untangling Continuous and Jump Components in Measuring, Modeling, and Forecasting Asset Return Volatility, Manuscript, Northwestern University, Duke University and University of Pennsylvania.

Andersen, T.G., T. Bollerslev, and F.X. Diebold, 2004, Parametric and Nonparametric Volatility Measurement, in L.P. Hansen and Y. Ait-Sahalia (eds.), Handbook of Financial Econometrics. Amsterdam: North-Holland, forthcoming.

Andersen, T.G., T. Bollerslev, F.X. Diebold and H. Ebens, 2001, "The Distribution of Realized Stock Return Volatility," Journal of Financial Economics, 61, 43-76.

Andersen, T., T. Bollerslev, F.X. Diebold, and P. Labys, 2000, Exchange Rate Returns Standardized by Realized Volatility are (Nearly) Gaussian, Multinational Finance Journal, 4, 159-179.

Andersen, T.G., T. Bollerslev, F.X. Diebold and P. Labys, 2001, "The Distribution of Realized Exchange Rate Volatility," Journal of the American Statistical Association, 96, 42-55.

Andersen, T.G., T. Bollerslev, F.X. Diebold, and P. Labys, 2003, Modeling and Forecasting Realized Volatility, Econometrica, 71, 529-626.

Andersen, T.G., T. Bollerslev, F.X. Diebold and C. Vega, 2004, Real-Time Price Discovery in Stock, Bond and Foreign Exchange Markets, Manuscript, Northwestern University, Duke University, University of Pennsylvania, and University of Rochester. 
Baillie, R.T. and T. Bollerslev, 1992, Prediction in Dynamic Models with Time-Dependent Conditional Variances, Journal of Econometrics, 51, 91-113.

Bandi, F. and J. Russell, 2004a, Separating Market Microstructure Noise from Volatility, Manuscript, University of Chicago.

Bandi, F. and J. Russell, 2004b, Microstructure Noise, Realized Volatility, and Optimal Sampling, Manuscript, University of Chicago.

Barndorff-Nielsen, O.E. and N. Shephard, 2001, Non-Gaussian Ornstein-Uhlenbeck-Based Models and Some of Their Uses in Financial Economics (with discussion), Journal of the Royal Statistical Society B, 63, 167-241.

Barndorff-Nielsen, O. and N. Shephard, 2004, Power and Bipower Variation with Stochastic Volatility and Jumps, Journal of Financial Econometrics, 2, 1-48.

Barone-Adesi, G., Bourgoin, F. and K. Giannopoulos, 1998, Don't Look Back, Risk, 11, August, 100-104.

Bates, D., 2000, Post-87 Crash Fears in S\&P 500 Futures Options, Journal of Econometrics 94, 181-238.

Benson, P. and P. Zangari, 1997, A General Approach to Calculating Var Without Volatilities and Correlations, RiskMetrics Monitor, 2nd Quarter, 19-23.

Berkowitz, J. and J. O'Brien, 2002, How Accurate are Value-at-Risk Models at Commercial Banks?, Journal of Finance, 57, 1093-1112.

Bodoukh, J., M. Richardson, and R. Whitelaw, 1998, The Best of Both Worlds, Risk, 11, May, 64-67.

Bollerslev, T., 1986, Generalized Autoregressive Conditional Heteroskedasticity, Journal of Econometrics, 31, 307-327.

Bollerslev, T., 1987, A Conditionally Heteroskedastic Time Series Model for Speculative Prices and Rates of Return, Review of Economics and Statistics, 69, 542-547.

Bollerslev, T., R.F. Engle, and J. Wooldridge, 1988, A Capital Asset Pricing Model with Time-Varying Covariances, Journal of Political Economy, 96, 116-131.

Bollerslev, T. and H.O. Mikkelsen, 1999, Long-Term Equity Anticipation Securities and Stock Market Volatility Dynamics, Journal of Econometrics, 92, 75-99.

Brandt, M., P. Santa-Clara and R. Valkanov, 2004, Optimal Portfolios with Parametric Weights, Manuscript, Duke University and UCLA. 
Cappiello, L., R.F. Engle and K. Sheppard, 2004, Asymmetric Dynamics in the Correlations of Global Equity and Bond Returns, Manuscript, New York University.

Chernov, M. and E. Ghysels, 2000, Towards a Unified Approach to the Joint Estimation of Objective and Risk Neutral Measures for the Purpose of Options Valuation, Journal of Financial Economics, 56, 407-458.

Christoffersen, P., 2003, Elements of Financial Risk Management. San Diego: Academic Press.

Christoffersen, P., S. Heston, and K. Jacobs, 2004, Option Valuation with Conditional Skewness, Journal of Econometrics, forthcoming .

Christoffersen, P., and K. Jacobs, 2004, Which GARCH Model for Option Valuation? Management Science, 50, 1204-1221.

Christoffersen, P., K. Jacobs, and Y. Wang, 2004, Option Valuation with Volatility Components, Manuscript, McGill University.

Diebold, F.X. A. Hickman, A. Inoue, and T. Schuermann, 1998, Converting 1-Day Volatility to h-Day Volatility: Scaling by Root-h is Worse than You Think, Wharton Financial Institutions Center, Working Paper 97-34. Published in condensed form as Scale Models, Risk, 11, 104-107.

Diebold, F.X. and M. Nerlove, 1989, The Dynamics of Exchange Rate Volatility: A Multivariate Latent-Factor ARCH Model, Journal of Applied Econometrics, 4, 1-22.

Diebold, F.X., T. Schuermann, and J. Stroughair, 1998, Pitfalls and Opportunities in the Use of Extreme Value Theory in Risk Management, in A.-P. N. Refenes, A.N. Burgess and J.D. Moody (eds.), Decision Technologies for Computational Finance, 3-12. Amsterdam: Kluwer Academic Publishers. (Reprinted in Journal of Risk Finance, 1 (Winter 2000), 30-36, and in P. Embrechts (ed.), Extremes and Integrated Risk Management. London: Risk Publications, 2000.)

Duffie, D. and J. Pan, 1997, An Overview of Value at Risk, Journal of Derivatives, Spring, 7-49.

Engle, R.F., 2001, GARCH 101: The Use of ARCH/GARCH Models in Applied Econometrics, Journal of Economic Perspectives, 15, 157-168.

Engle, R.F., 2002, Dynamic Conditional Correlation: A Simple Class of Multivariate Generalized Autoregressive Conditional Heteroskedasticity Models, Journal of Business and Economic Statistics, 20, 339-350.

Engle, R.F., 2004, Risk and Volatility: Econometric Models and Financial Practice, American Economic Review, 94, 405-420. 
Engle, R.F. and G.M. Gallo, 2004, A Multiple Indicators Model for Volatility Using Intra-Daily Data, Manuscript, New York University and University of Firenze.

Engle, R.F. and G.G.J. Lee, 1999, A Permanent and Transitory Component Model of Stock Return Volatility, in R.F. Engle and H. White (eds.), Cointegration, Causality, and Forecasting: A Festschrift in Honor of Clive W.J. Granger, 475-497. Oxford, UK: Oxford University Press.

Engle, R.F. and J. Mezrich, 1996, GARCH for Groups, Risk, 8, 36-40.

Eraker, B., 2004, Do Stock Prices and Volatility Jump? Reconciling Evidence from Spot and Option Prices, Journal of Finance, 59, 1367-1403.

Fernandez, C. and M.F.J. Steel, 1998, On Bayesian Modeling of Fat Tails and Skewness, Journal of the American Statistical Association, 93, 359-371.

Fleming, J. C. Kirby, and B. Ostdiek, 2003, The Economic Value of Volatility Timing Using Realized Volatility, Journal of Financial Economics, 67, 473-509.

Franses, P.H and C. Hafner, 2003, A Generalized Dynamic Conditional Correlation Model for Many Asset Returns, Manuscript, Erasmus University Rotterdam.

Gallant, A.R., C.T. Hsu, and G.E. Tauchen, 1999, Using Daily Range Data to Calibrate Volatility Diffusions and Extract the Forward Integrated Variance, Review of Economics and Statistics, 81, 617-631.

Ghysels, E., P. Santa-Clara and R. Valkanov, 2005, Predicting Volatility: Getting the Most out of Return Data Sampled at Different Frequencies, Journal of Econometrics, forthcoming.

Glasserman, P., P. Heidelberger, and P. Shahbuddin, 2002, Portfolio Value-at-Risk with Heavy-tailed Risk Factors, Mathematical Finance, 239-269.

Glosten, L.R., R. Jagannathan, and D. Runkle, 1993, On the Relation Between the Expected Value and the Volatility of the Nominal Excess Return on Stocks, Journal of Finance, 48, 1779-1801.

Guidolin, M. and A. Timmermann, 2004, Term Structure of Risk under Alternative Econometric Specifications, Journal of Econometrics, forthcoming.

Hansen, B., 1994, Autoregressive Conditional Density Estimation, International Economic Review, 35, 705-730.

Hansen, P.R. and A. Lunde, 2004, A Realized Variance for the Whole Day Based on Intermittent High-Frequency Data, Manuscript, Stanford University. 
Hartmann, P., S. Straetmans, and C. de Vries, 2004, Banking System Stability: A Cross-Atlantic Perspective, forthcoming as a chapter in this volume.

Harvey, A.C., 1989, Forecasting Structural Time Series Models and the Kalman Filter, Cambridge University Press.

Harvey, A.C., E. Ruiz, and E. Sentana, 1992, Unobserved Component Time Series Models with ARCH Disturbances, Journal of Econometrics, 52, 129-157.

Heston, S., 1993, A Closed-Form Solution for Options with Stochastic Volatility with Applications to Bond and Currency Options, Review of Financial Studies 6, 327-343.

Heston, S. and S. Nandi, 2000, A Closed-Form GARCH Option Pricing Model, Review of Financial Studies 13, 585-626.

Hull, J., and A. White, 1998, Incorporating Volatility Updating into the Historical Simulation Method for VaR, Journal of Risk 1, 5-19.

Jackson, P., D. Maude and W. Perraudin, 1997, Bank Capital and Value at Risk, Journal of Derivatives, 4, 73-89.

Jondeau, E. and M. Rockinger, 2004, The Copula-GARCH Model of Conditional Dependence: An International Stock Market Application, Journal of International Money and Finance, forthcoming.

Jorion, P., 2000, Value-at-Risk. New York: McGraw-Hill.

Ledoit, O., P. Santa-Clara, and M. Wolf, 2003, Flexible Multivariate GARCH Modeling with an Application to International Stock Markets, Review of Economics and Statistics, 85, 735747.

Ledoit, O., and M. Wolf, 2003, Improved Estimation of the Covariance Matrix of Stock Returns with an Application to Portfolio Selection, Journal of Empirical Finance, 10, 603-621.

Longin, F. And B. Solnik, 2001, Extreme Correlation of International Equity Markets, Journal of Finance, 56, 649-676.

Manganelli, S., 2004, Asset Allocation by Variance Sensitivity Analysis, Journal of Financial Econometrics, 2, 370 - 389.

Martens, M., 2004, Estimating Unbiased and Precise Realized Covariances, Manuscript, Erasmus University Rotterdam.

McNeil, A.J. and R. Frey, 2000, Estimation of Tail-Related Risk Measures for Heteroskedastic Financial Time Series: An Extreme Value Approach, Journal of Empirical Finance, 7, 271-300. 
Nerlove, M. and S. Wage, 1964, On the Optimality of Adaptive Forecasting, Management Science, 10, 207-229.

Pan, J., 2002, The Jump-Risk Premia Implicit in Options: Evidence from an Integrated TimeSeries Study, Journal of Financial Economics, 63, 3-50.

Patton, A.J., 2002, Modeling Time-varying Exchange Rate Dependence Using the Conditional Copula. Working paper, LSE.

Pelletier, D., 2004, Regime Switching for Dynamic Correlations, Journal of Econometrics, forthcoming.

Pesaran, H., and P. Zaffaroni, 2004, Model Averaging and Value-at-Risk based Evaluation of Large Multi Asset Volatility Models for Risk Management, Manuscript, University of Cambridge.

Poon, S.-H., M. Rockinger, and J. Tawn, 2004, Extreme Value Dependence in Financial Markets: Diagnostics, Models and Financial Implications, Review of Financial Studies, 17, 581-610.

Pritsker, M., 2001, The Hidden Dangers of Historical Simulation, Manuscript, Federal Reserve Board.

Scholes, M., and J.T. Williams, 1977, Estimating Betas from Nonsynchronous Data, Journal of Financial Economics, 5, 309-327.

Theil, H., and S. Wage, 1964, Some Observations on Adaptive Forecasting, Management Science, 10, 198-206.

Zhou, B., 1996, "High-Frequency Data and Volatility in Foreign-exchange Rates, Journal of Business and Economic Statistics, 14, 45-52. 
Figure 1. October 1987: Daily S\&P500 Loss and 1\% HS-VaR

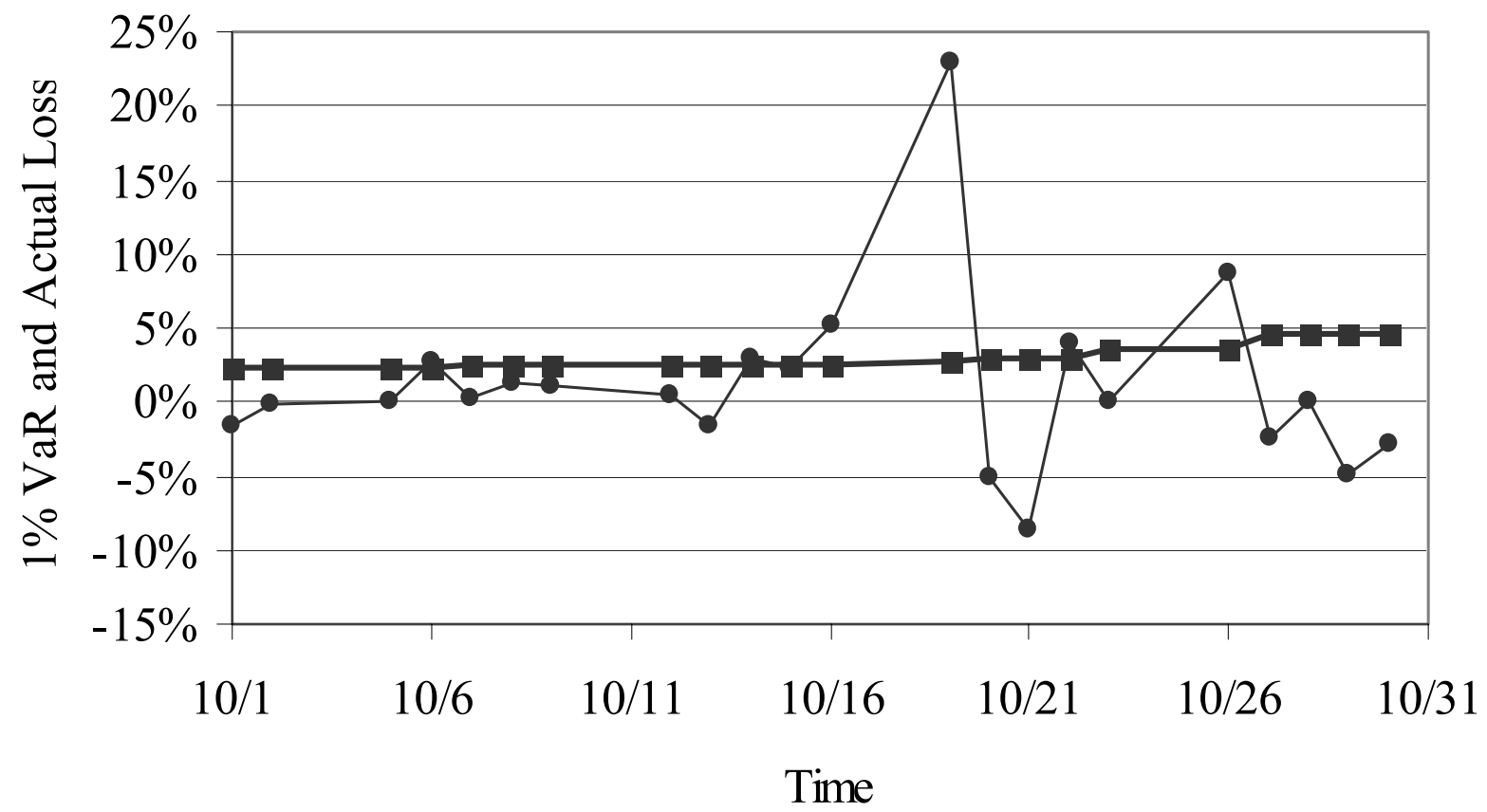

Notes to Figure: The thin line with diamonds shows the daily percentage loss on an S\&P500 portfolio during October 1987 . The thick line with squares shows the daily $1 \% \mathrm{VaR}$ from historical simulation using a 250-day window. 
Figure 2. True Conditional Coverage of $1 \%$ VaR from Historical Simulation

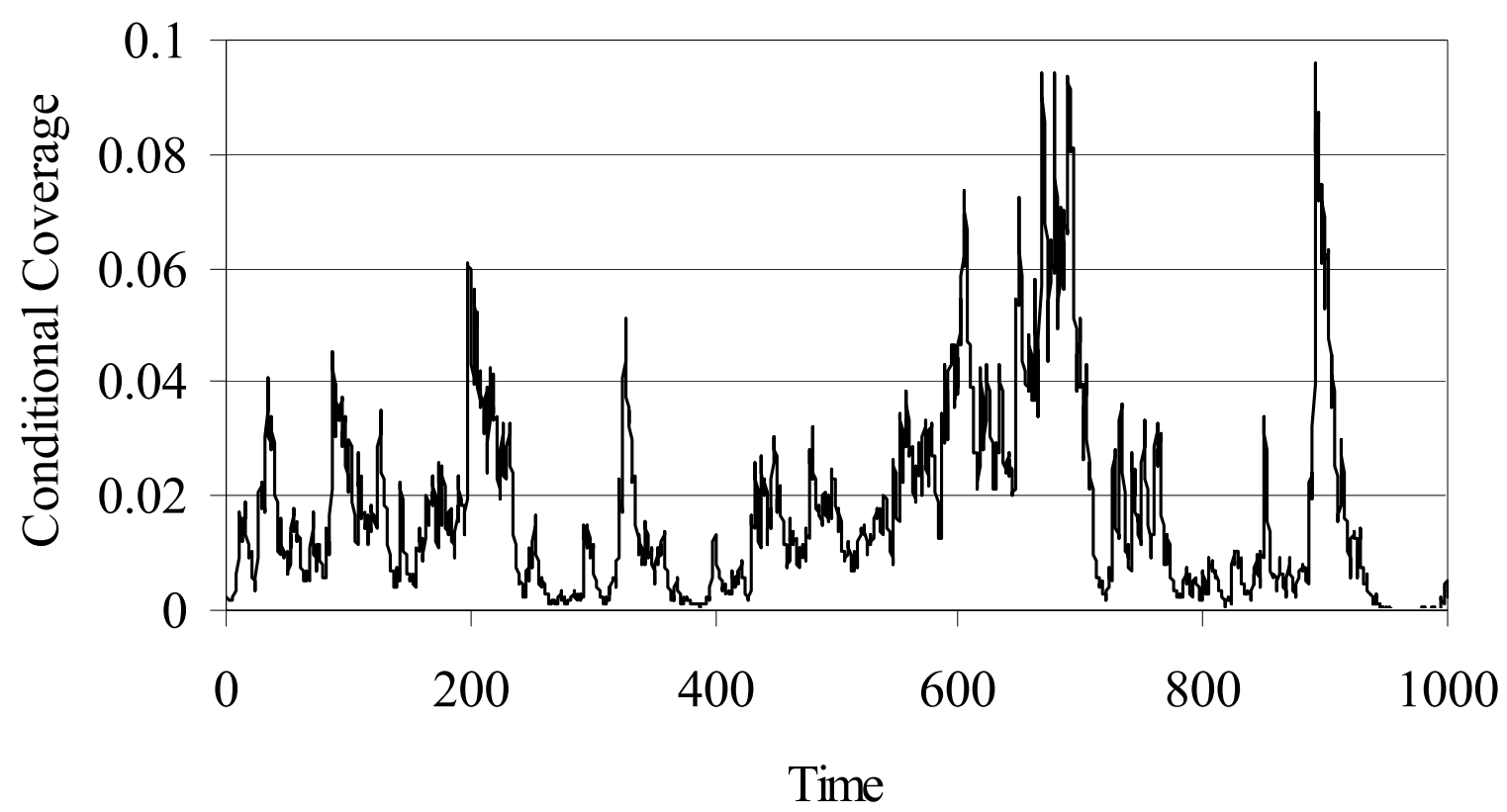

Notes to Figure: We simulate returns from a GARCH model with normal innovations, after which we compute the $1 \% \mathrm{HS}-\mathrm{VaR}$ using a rolling window of 250 observations, and then we plot the true conditional coverage probability of the HS-VaR, which we calculate using the GARCH structure. 
Figure 3. Term Structure of Variance in GARCH and RiskMetrics Models

Notes to Figure: We plot the term structure of variance from a mean-reverting GARCH model (thick line) as well as the term structure from a RiskMetrics model (thin line). The current variance is assumed to be identical across models.

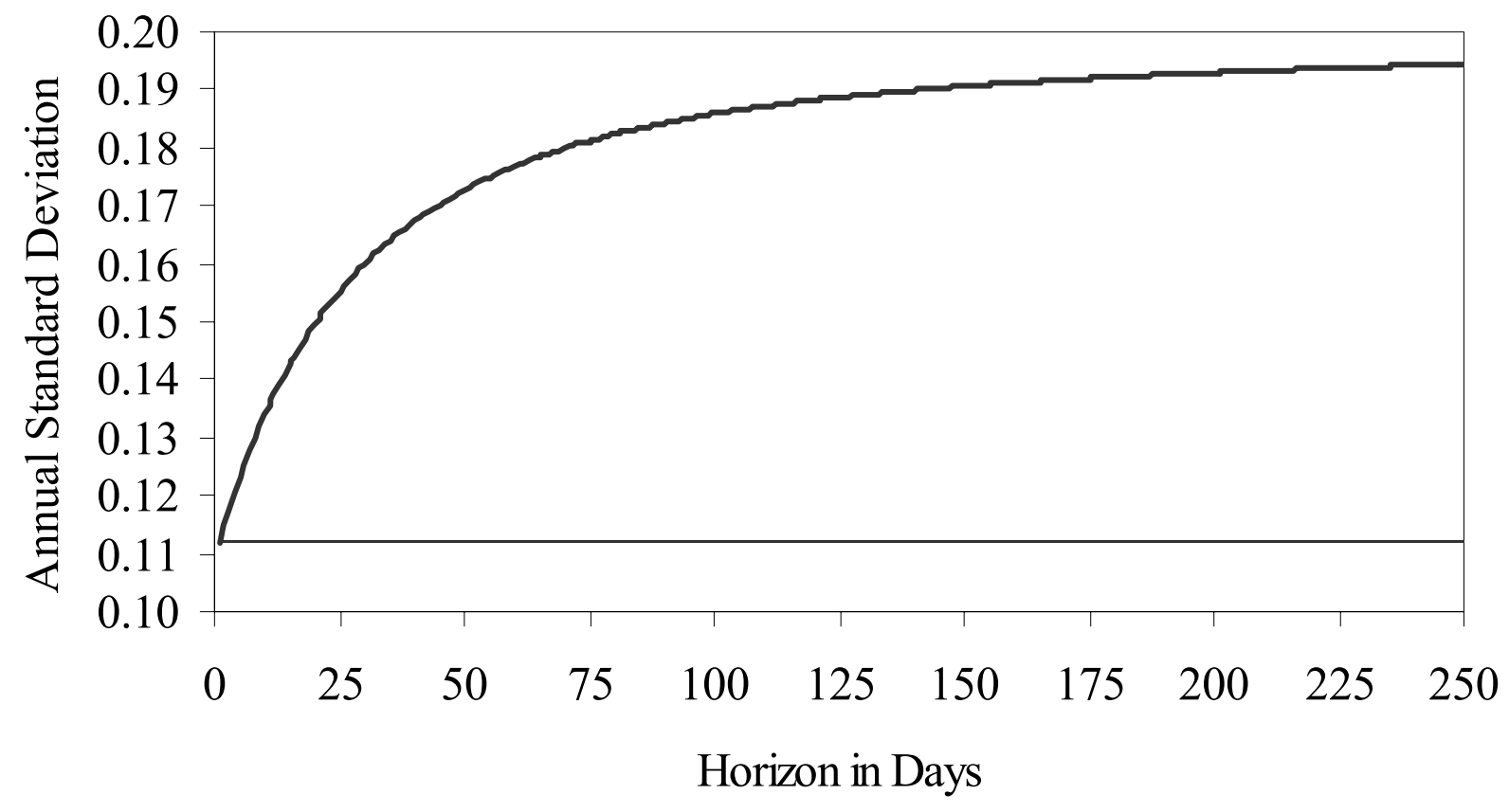


Figure 4. Time-Varying Bond Return Correlation: Germany and Japan

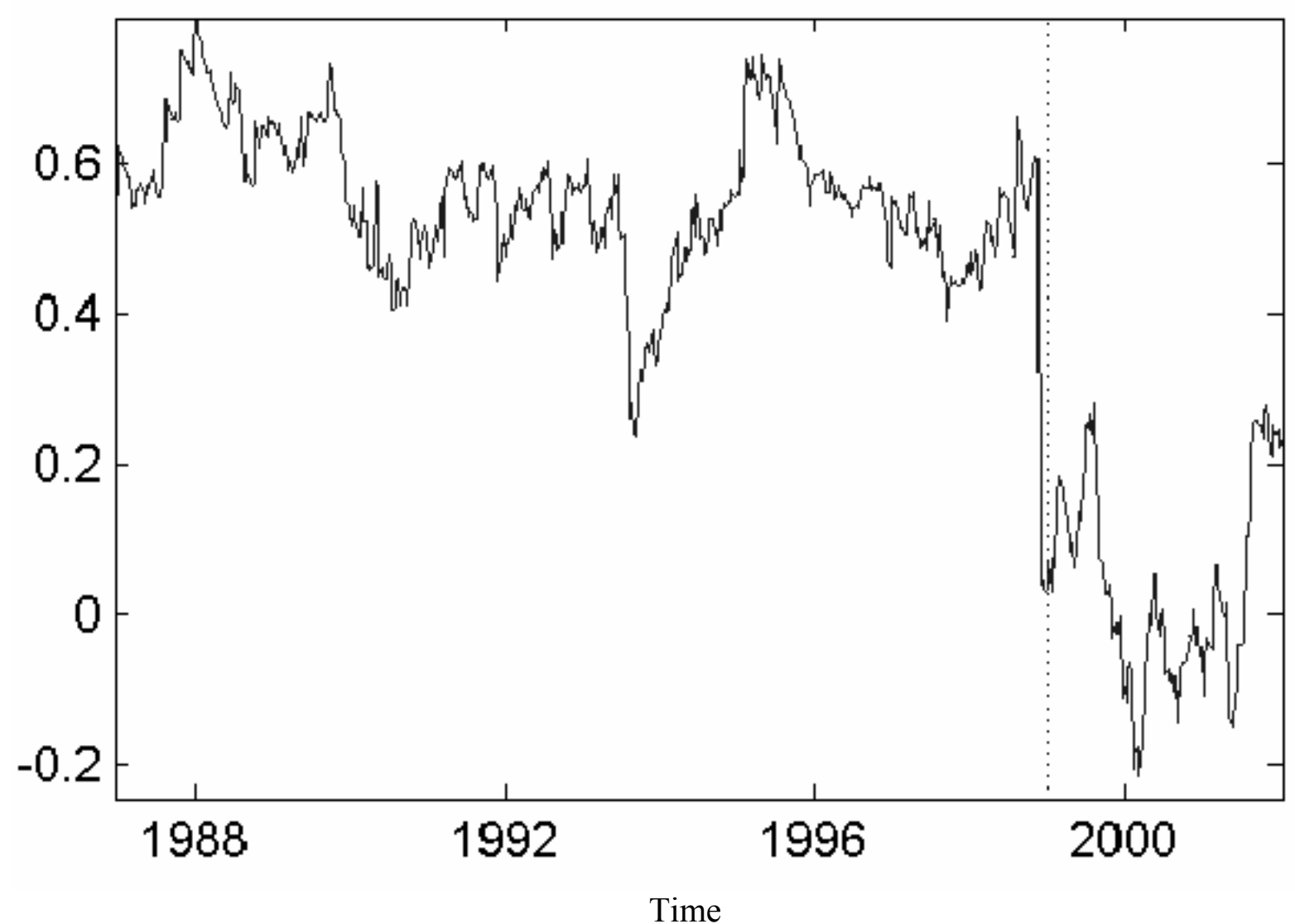

Notes to Figure: We reconstruct this figure from Capiello, Engle and Sheppard, 2004, plotting the correlation between German and Japanese government bond returns calculated from a DCC model allowing for asymmetric correlation responses to positive and negative returns. The vertical dashed line denotes the Euro's introduction in 1999. 
Figure 5. Sample Autocorrelations of Realized Volatility: Three Currencies

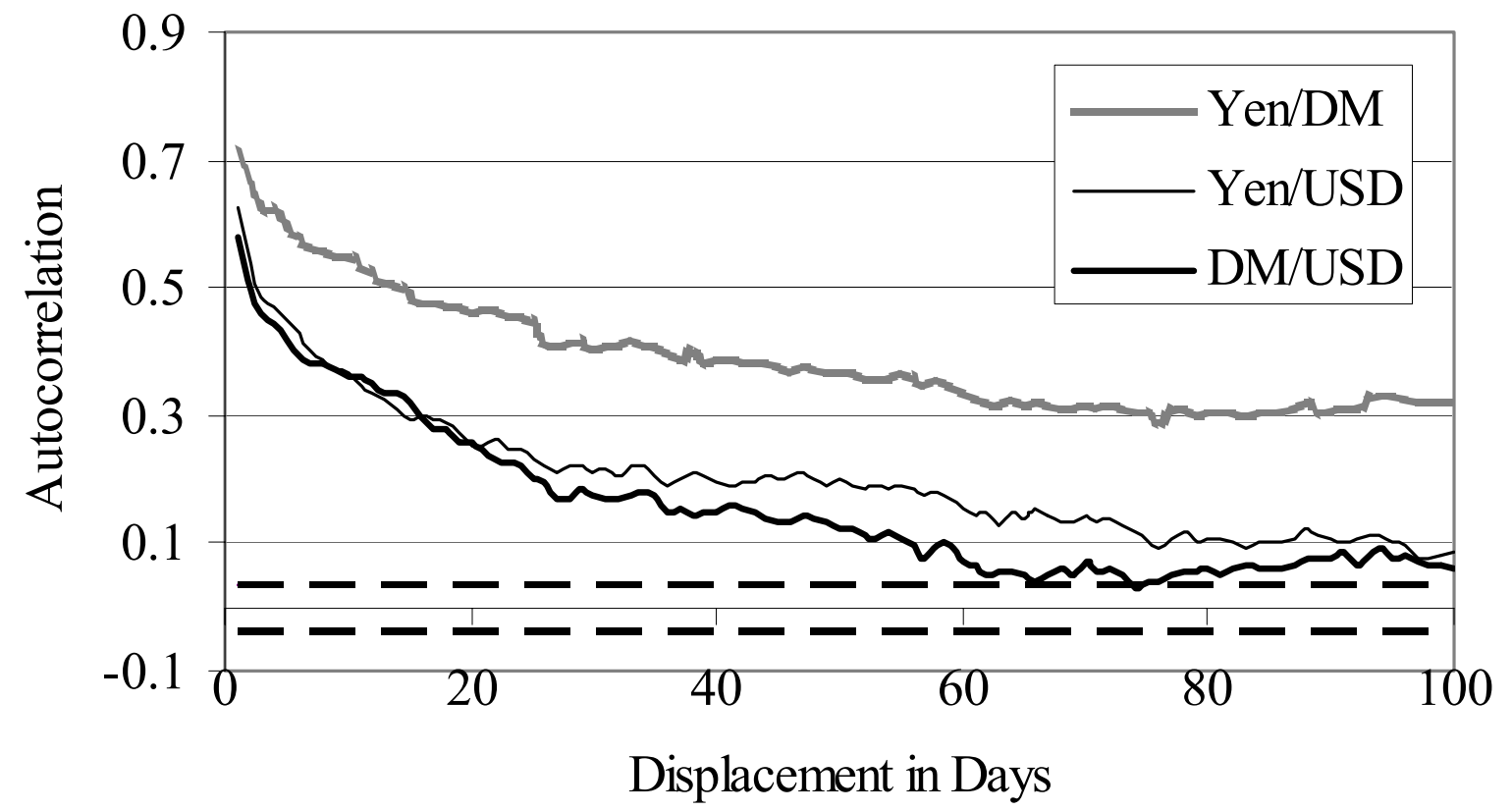

Notes to Figure: We plot the sample autocorrelations of daily realized log standard deviations for three FX rates, together with Bartlett's +/- 2 standard error bands for the sample autocorrealtions of white noise. We construct the underlying daily realized variances using 30-minute returns from December 1, 1986, through December 1, 1996. 
Figure 6. QQ Plot of S\&P500 Returns Standardized by the Average Volatility

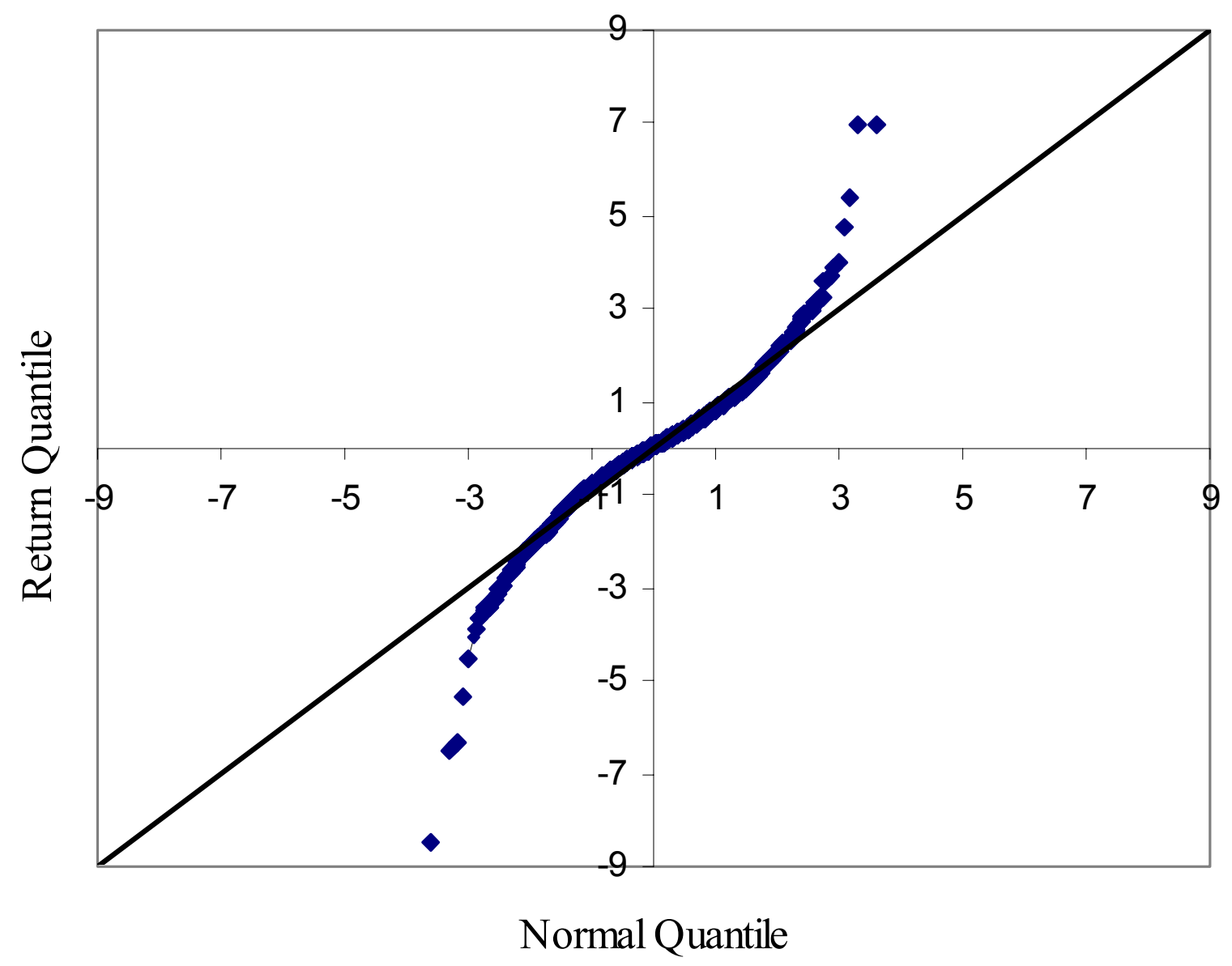

Notes to Figure: We show quantiles of daily S\&P500 returns from January 2, 1990 to December 31,2002 , standardized by the average daily volatility during the sample, against the corresponding quantiles from a standard normal distribution. 
Figure 7. QQ Plot of S\&P500 Returns Standardized by GARCH Volatility

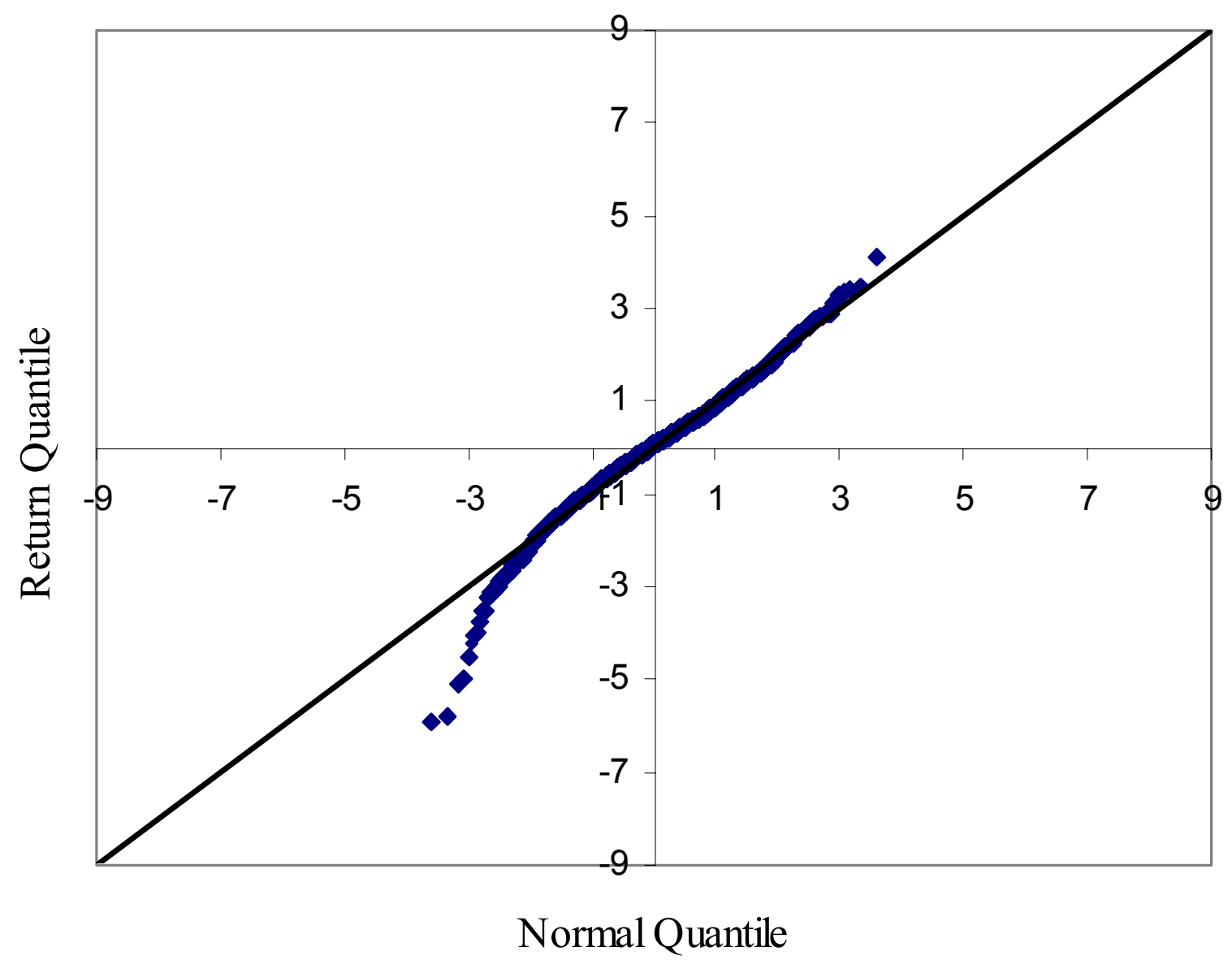

Notes to Figure: We show quantiles of daily S\&P500 returns from January 2, 1990 to December $31,2002$, standardized by volatility from a estimated asymmetric GJR GARCH( 1,1$)$ model, against the corresponding quantiles from a standard normal distribution. 
Figure 8. QQ Plot of S\&P500 Returns Standardized by Realized Volatility

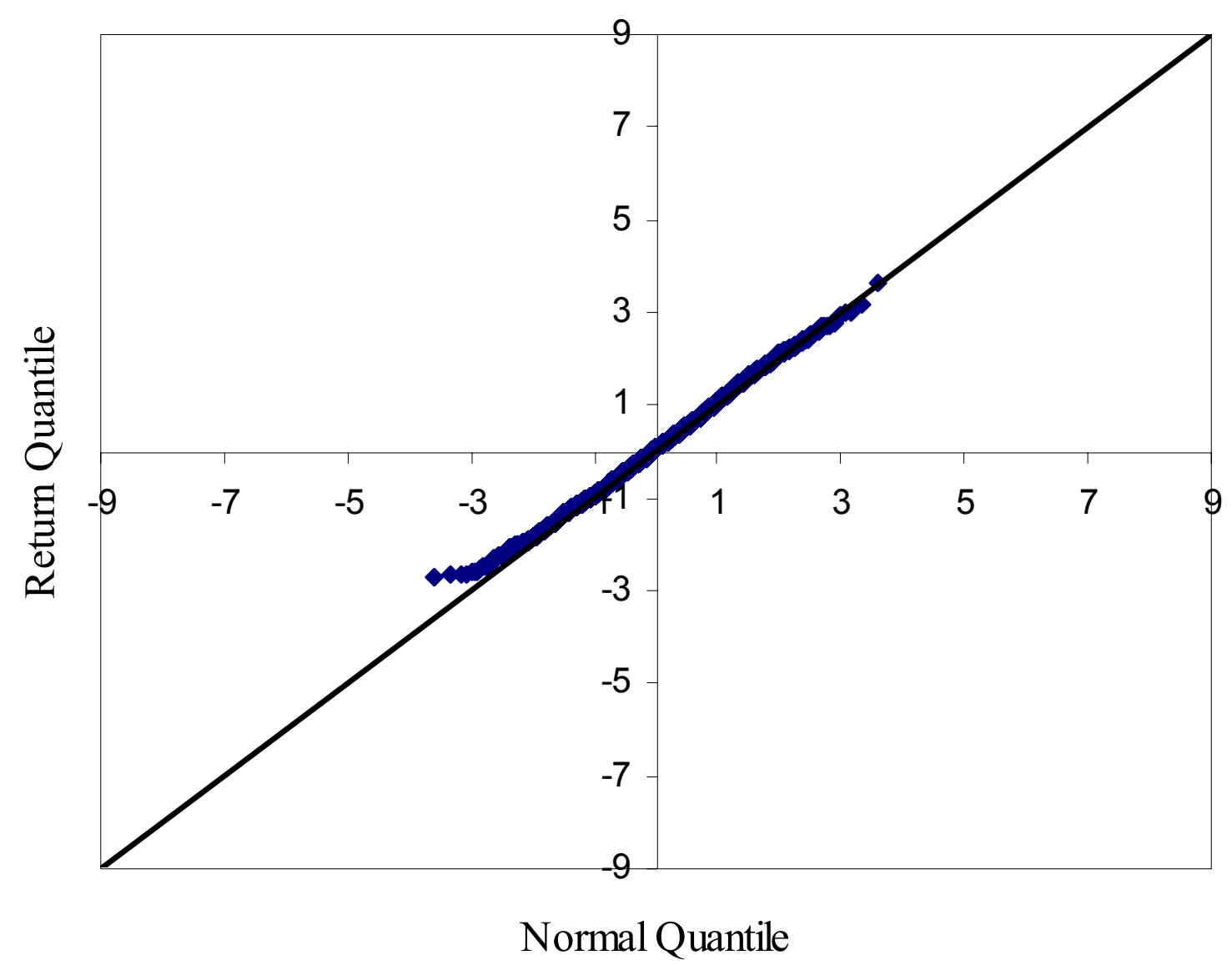

Notes to Figure: We show quantiles of daily S\&P500 returns from January 2, 1990 to December 31,2002 , standardized by realized volatility calculated from 5-minute futures returns, against the corresponding quantiles from a standard normal distribution. 